\title{
Characterization of liquid-liquid flows in horizontal pipes
}

\author{
Jing Shi and Hoi Yeung \\ Oil and Gas Engineering Centre, School of Energy, Environment and Agrifood, \\ Cranfield University, Cranfield MK43 OAL, UK
}

\begin{abstract}
Diverse flow regimes have been encountered in liquid-liquid flows. Some degree of consistency in the observed flow patterns is shown in reported studies, while inconsistency exits when physical properties of the two phases concerned are wide enough. An attempt was made in this study to investigate the mechanisms behind flow patterns of liquid-liquid flows in horizontal pipes. A literature review on flow patterns of liquid-liquid flows in horizontal pipes was conducted. The ratio of the gravitational force to viscous force was proposed to characterize liquid-liquid flows in horizontal pipes into gravitational force dominant, viscous force dominant and gravitational force and viscous force comparable flow featured with different basic flow regimes. Comparisons of the proposed characterization criterion with the literature data show good agreement.
\end{abstract}

Keywords: liquid-liquid flows; flow patterns; characterization; gravitational force; viscous force.

Correspondence concerning this article should be addressed to J. Shi at ingshi1988@gmail.com or H. Yeung at h.yeung@cranfield.ac.uk 


\section{Introduction}

Flows of immiscible liquids are encountered in a diverse range of processes. In the petroleum industry, oil and water are frequently transported together in wells and pipelines. A lot of studies on particular liquid-liquid flow systems, e.g., low-viscosity oil-water flow ${ }^{1-8}$, highviscosity oil-water flow ${ }^{9-21}$, specific flow regimes such as stratified flow ${ }^{22,23}$, dispersed flow $24-28$ and core annular flow ${ }^{9-11}, 29-36$, have been reported in the literature. These studies greatly contribute to our understanding of liquid-liquid flows. Brauner ${ }^{37}$ conducted a comprehensive review on liquid-liquid two-phase flow. It was summarized that the diverse flow patterns can be classified into four basic prototypes: stratified layers with either smooth or wavy interface; large slugs, elongated or spherical, of one liquid in the other; a dispersion of relatively fine drops of one liquid in the other; and annular flow, where one of the liquids forms the core and the other liquid flows in the annulus. In many cases, the flow pattern consists of a combination of the aforementioned basic prototypes and can be regarded as transitional patterns. The flow regime maps produced from different studies are not always consistent. Some flow maps are comparable with same basic flow patterns and similar transition trends, while some other flow maps are not comparable with different basic flow regimes. Mechanisms which govern different liquid-liquid flows featured with different basic flow patterns are not well established.

In this article, characteristics of liquid-liquid flows in horizontal pipes and mechanisms behind the flow characteristics are investigated. In the section Liquid-Liquid Flow Patterns in Horizontal Pipes - Literature Review, a survey on flow regimes of liquid-liquid flows in horizontal pipes (5.6 mm $\leq$ I.D. $\leq 200 \mathrm{~mm}, 1.3 \leq \mu_{\mathrm{o}} / \mu_{\mathrm{w}} \leq 91600$ ) is described. In the section Experimental Campaign and Results, a brief introduction on our experimental campaign is presented followed by a discussion of experimental results. In the section Characterization of Liquid-Liquid Flows in Horizontal Pipes, the underlying physics which govern the formation of different basic flow regimes of liquid-liquid flows are discussed. The ratio of the gravitational force to viscous force is proposed to characterize liquid-liquid flows featured with different basic flow regimes. Finally, the section Conclusions presents the major conclusions. 


\section{Liquid-Liquid Flow Patterns in Horizontal Pipes - Literature Review}

\section{Low-viscosity oil-water flow}

Most studies - stratified and dispersed flow

Trallero et al. ${ }^{1}$ reviewed flow patterns of low-viscosity $\left(1.3 \leq \mu_{0} / \mu_{\mathrm{w}} \leq 167\right)$, unequal-density $\left(0.75 \leq \rho_{\mathrm{o}} / \rho_{\mathrm{w}} \leq 0.9\right)$ oil-water flow in horizontal pipes with internal diameters ranging from 24 to $59 \mathrm{~mm}$. He proposed six flow patterns in two basic categories - segregated flow (ST, ST \& MI) and dispersed flow (Do/w \& w, Do/w, Dw/o \& Do/w, Dw/o). Same flow regimes were reported by Vielma et al. ${ }^{2}$ Two more specific flow patterns - Dw/o \& w and Dw/o \& Do/w \& w, which can be categorised into the dispersed flow, were reported by Nädler and Mewes. ${ }^{3}$ Figure 1 shows sketches of these flow patterns. Nädler and Mewes $^{3}$ also observed that there was little effect of viscosity on the flow characteristics from measurements conducted for oil viscosities of 22, 27 and $35 \mathrm{mPa}$.s. Angeli and Hewitt ${ }^{4}$ studied flow patterns in 1 inch stainless steel and acrylic resin pipes respectively. Though different nomenclatures were used by the above authors, flow patterns observed are consistent with the flow patterns depicted in Figure 1. A flow regime of Three Layer (3L) was proposed to describe flow in which a mixed layer is present between the water and oil layers at the bottom and top of the pipe respectively. This flow regime corresponds to ST \& MI when the mixing at the interface is intense leading to a thick mixing layer. They also found that flow patterns in the steel pipe were in general more disturbed (i.e., having a wider fully dispersed region) than those in the acrylic pipe and attributed this to the higher roughness of the steel pipe. Lovick and Angeli ${ }^{5}$ used the term of dual continuous flow (both phases retain their continuity at the top and bottom of the pipe while there is interspersion) to describe transition between stratified flow and dispersions of one phase in another phase. Following this classification, dual continuous flow includes ST \& MI and Dw/o \& Do/w defined by Trallero et al. ${ }^{1}$, Dw/o \& w and Dw/o \& Do/w \& w by Nädler and Mewes $^{3}$ and $3 \mathrm{~L}$ by Angeli and Hewitt ${ }^{4}$.

\section{(Figure 1)}

Most experimental studies on low-viscosity oil-water flow in the literature show consistence in flow patterns. Stratified flow and dispersed flow are two basic phase configurations; 
transition between the basic flow regimes leads to more specific flow structures. However, there are a few low-viscosity oil-water studies in which different flow patterns were observed as presented in the following two sections.

Density-matched oil-water flow - annular, intermittent, and dispersed flow

Different flow characteristics are shown for density-matched oil-water flow. Charles et al. ${ }^{6}$ investigated equal-density oil-water flow in a horizontal 1 inch pipe. Oils with viscosities of 6.29, 16.8 and $65.0 \mathrm{mPa} \cdot \mathrm{s}$ were used in the experiments. Five flow patterns, namely dispersed oil drops in water (Do/w), core annular flow (CAF), oil slugs in water (OSL), large oil drops in water (ODR) or oil bubbles in water as termed by the authors, and dispersed water droplets in oil (Dw/o), were observed. OSL and ODR can be grouped into intermittent flow. These phase configurations are shown in Figure 2. Compared to the flow patterns of low-viscosity unequal-density oil-water flow introduced above, the common flow patterns are Do/w and Dw/o. The annular and intermittent flow regimes were newly observed flow patterns while stratified flow was not observed. It's apparent that this significant difference in flow structures comes from the equal-density of the two phases. Without the difference of gravitational forces, the two phases do not become stratified. When the turbulence kinetic energy of the two immiscible liquids is not sufficient to cause the formation of dispersed flow, the flow exists in forms of one phase flowing forward, continuously (i.e., CAF) or discontinuously (i.e., OPL and ODR), inside the other phase.

\section{(Figure 2)}

Flow in small diameter pipes - stratified, annular, intermittent, and dispersed flow

The flow patterns of oil-water flow in small diameter pipes also show difference from those observed in most studies on low-viscosity oil-water flow. It is difficult to define a size range for the so-called small diameter pipes. Normally the pipe I.D. is smaller than 1 inch and larger than $2 \mathrm{~mm}$ when the term of small diameter is used. ${ }^{38,39}$

Russell et al. ${ }^{7}$ conducted experiments on horizontal oil-water flow in a $20.5 \mathrm{~mm}$ I.D. pipe. The viscosity of the oil used in their experiments is $16.8 \mathrm{mPa} \cdot \mathrm{s}$. Stratified, intermittent (oil slugs/plugs, large oil drops in water), and dispersed flow were observed. Though this study 
was among the studies surveyed by Trallero et al. ${ }^{1}$ in their review on low-viscosity oil-water flow patterns, the intermittent flow did not draw the above authors' attention since all the other studies in their survey reported exclusively stratified and dispersed flow.

Wegmann and Rudolf von Rohr ${ }^{8}$ conducted experiments on horizontal oil-water flow in pipes with internal diameters of 5.6 and $7 \mathrm{~mm}$. The viscosity of the paraffin oil used in their experiments varied from 4.3 to $5.2 \mathrm{mPa} \cdot \mathrm{s}$. They observed stratified, intermittent (oil slugs/plugs in water), annular, and dispersed flow. Dispersions of water in the oil core of annular flow were observed. A comparison was made between the flow pattern map produced from their experiments to that produced by Angeli and Hewitt ${ }^{4}$. As one can expect, a large difference between the flow pattern maps was shown since the basic flow patterns observed in the two studies are not consistent.

In general, except for stratified flow and dispersed flow, intermittent flow (oil slugs/plugs, large oil drops in water) and/or core annular flow are encountered for low-viscosity unequaldensity oil-water flow in small diameter horizontal pipes.

\section{High-viscosity oil-water flow}

Since the experiment of Charles et al. ${ }^{6}$, experiments on high-viscosity oil-water flow have been carried out due to the great potential of core annular flow in transporting heavy crude oil. Ooms et al. ${ }^{9}$ conducted experiments on high-viscosity oil-water flow in horizontal pipes. Oil viscosities investigated by the above authors varied from 2300 to $3300 \mathrm{mPa} \cdot \mathrm{s}$ in a 2 inch Perspex pipe of $50 \mathrm{~m}$ length and 1200 to $2200 \mathrm{mPa} \cdot \mathrm{s}$ in an 8 inch pipe of $888 \mathrm{~m}$ length. With a focus on CAF, only CAF was reported without information of other flow structures formed in their experiments. Studies focused on CAF can also be found in Oliemans et al. ${ }^{10}$ and Bannwart ${ }^{11}$. Arney et al. ${ }^{12}$ conducted experiments on high-viscosity oil-water flow in a horizontal pipe (I.D.=16 mm) using waxy crude oil (stable water-in-oil emulsion) and No. 6 fuel oil (viscosity $2700 \mathrm{mPa} \cdot \mathrm{s}$ ). The test section was a glass pipe of $6.35 \mathrm{~m}$ length. Core annular flow with oil fouling clots on the pipe wall, termed as 'oil sticks to the wall' by the above authors was observed at low input water volume fraction. With increase of the input water volume fraction, nearly perfect core annular flow, wavy core annular flow, and oil plugs in water were 
observed. It is noted that the above authors also reported that originally a transparent PVC pipe was used. The pipeline was cleaned regularly with detergent and water. This method worked well with the waxy crude oil, but not for the No. 6 fuel oil. The No. 6 fuel oil tended to adhere to the wall of the PVC pipe and the oil clots formed could not be cleaned from the pipe wall with detergent and water. The PVC pipe section was changed to the glass pipe as the glass was preferentially wetted by water. Oil fouling on the wall of the glass pipe could be removed by running clean water through the pipe. McKibben et al. ${ }^{13,14}$ investigated heavy oilwater flows in horizontal steel pipelines with short glass sections for visual observation. Laboratory experiments were conducted in pipelines with internal diameters of 53 and 105 mm using high-viscosity lube oil and crude oil. They found that at low mixture velocities and/or low water fractions there exists an intermittent phase where water intermittently reduces the pressure gradient of heavy oil flow and associated this phase with water slugs which envelop some oils. They also observed continuous water-assisted flow with oil fouling layer on the internal pipe wall at high velocities and/or water fractions. Apart from the laboratory experiments, McKibben et al. ${ }^{14}$ also conducted field tests in a filed pipeline (I.D. $=80.4 \mathrm{~mm}$; length $1336 \mathrm{~m}$ ). Core annular flow with oil fouling layer on the pipe wall, or continuous waterassisted flow as termed by the above authors, was found to be achieved provided the water fraction and the mixture velocity were high enough. Bannwart et al. ${ }^{15}$ reported flow patterns of heavy oil-water flow in experiments carried out in a flow loop comprising both vertical and horizontal test sections (glass pipe, I.D.=28.4 mm). The oil (viscosity $488 \mathrm{mPa} \cdot \mathrm{s}$ ) and water were introduced concentrically via an injection nozzle. Basic flow patterns observed in the horizontal pipe were core annular flow, large oil drops in water, and dispersed oil lumps in water. Grassi et al. ${ }^{16}$ conducted experiments on oil-water flow in both horizontal and slightly inclined (up to $\pm 15^{\circ}$ ) polycarbonate pipes (I.D.=21 mm) using oil with a viscosity around 800 $\mathrm{mPa} \cdot \mathrm{s}$. Core annular flow and dispersed oil in water were found to be the major flow patterns; oil slugs/plugs in water and stratified flow were also observed under a few limited flow conditions. No substantial differences were found with change in inclination angles. It should be noted that the specific phase configuration of dispersed oil in water for high-viscosity oil- 
water flow is different from that for low-viscosity oil-water flow in the degree of dispersion. Emulsion or fine dispersed drops of one phase in the other phase is developed in low-viscosity oil-water flow, while the dispersed oil in water defined by Grassi et al. ${ }^{16}$ shows the characteristic that the oil is dispersed into lumps with varying sizes. Sotgia et al. ${ }^{17}$ reported their experimental investigation on oil-water flow in horizontal pipes of Plexiglas and Pyrex (I.D. varies from 21 to $40 \mathrm{~mm}$ ) using oil with a viscosity around $900 \mathrm{mPa} \cdot \mathrm{s}$. Flow patterns observed are similar to those reported by Grassi et al. ${ }^{16}$ Sridhar et al. ${ }^{18}$ conducted experiments on high-viscosity oil-water flow in horizontal and slightly inclined steel pipelines. The oil used has a viscosity range from 200 to $1100 \mathrm{mPa} \cdot \mathrm{s}$. The basic two flow structures observed are stratified flow and core annular flow. More specific flow nomenclatures were used by the authors depending on whether there were dispersed oil drops at the interface and whether oil fouling film on the pipe wall was observed. Al-Awadi ${ }^{19}$, Alagbe ${ }^{20}$, and $\mathrm{Shi}^{21}$ studied flow characteristics of high-viscosity oil-water flow in a horizontal Perspex pipe (I.D. $=26 \mathrm{~mm}$ ) with oil viscosity between 3300 to $16000 \mathrm{mPa} \cdot \mathrm{s}$. A transitional flow regime between oil-continuous and water-continuous flow characterized with spiral motion of water and oil was observed. Specific flow structures in water-continuous flow were further classified into core annular flow, oil plugs in water and dispersed oil lumps in water.

In general, CAF is a dominant flow pattern developed in a wide range of phase flow rates in high-viscosity oil-water flows. Intermittent (oil slug/plugs, large oil drops in water) and dispersed oil in water are usually encountered. Oil dispersion in water is different from Do/w emulsions in views of irregularity and variety in the shape and size of the dispersed phase, and the instability of the mixture. Stratified flow is reported for limited flow conditions. Specific flow structures in oil-continuous flow are less reported due to reduced region of oil-continuous flow, difficulty in visual observation for oil-continuous flow as well as more interest in watercontinuous flow. Figure 3 shows sketches of most frequently reported water-continuous flow patterns in horizontal high-viscosity oil-water pipeline flow. The sketches at the left and right hand of the figure illustrate the phase configurations without and with oil fouling on the pipe internal wall, respectively. As introduced above, oil fouling film on the inner wall of the pipe 
was observed in some experiments in a wide range of phase flow rates ${ }^{13,14,18-21}$ or in limited flow rates ${ }^{12,16}$, while not observed (or not reported due to different focus of interest) in some other experiments ${ }^{9-11,15,17}$. This is much likely to be associated with the wettability of the pipe wall to the fluids (i.e., pipe material, wall roughness and fluids property) and the experimental method and procedure (e.g., whether special injection device is used to favor the formation of $\mathrm{CAF}$ at the injection point; whether water/detergent is regularly used to wash the pipe; the sequence of the phase injection; and the experimental run time). For the above experiments conducted in glass pipes which are normally hydrophilic and have a small degree of wall roughness, oil fouling was not reported ${ }^{15}$ or only observed at limited flow conditions ${ }^{12}$. Also noted is that special care was taken in those experiments to reduce the chance of oil contact to the pipe wall, e.g., introducing the fluids into the pipe in the form of core annular flow via an injection device. For the experiments conducted in steel pipelines ${ }^{13,14,18}$, oil fouling on the pipe wall was observed despite the fact that clean steel pipes are normally hydrophilic ${ }^{40}$. This might be related to a higher degree of wall roughness for steel pipes, the use history of the pipes such as being used to transport oil and the experimental procedure. For experiments conducted in Perspex or other types of plastic pipes which are normally hydrophobic, oil fouling was not observed/reported in some studies ${ }^{9,10,17}$, while different degree of oil fouling was observed in some other studies ${ }^{16,19-21}$. Differences in the degree of the wall wettability to the fluids and the experimental procedure (whether special procedures was used to reduce the possibility of fouling) can be the sources of different observations. For industrial operations of water-lubricated heavy oil transport, oil fouling on the pipe wall is inevitable. The hydrodynamic stability of water-lubricated flow is robust even when oil wets the wall. ${ }^{30}$ Core annular flow with oil fouling on the pipe wall is stable in certain flow conditions as shown in laboratory experiments ${ }^{13,14,19-21}$ and field tests ${ }^{14}$. For stable CAF with oil fouling, the height of the oil fouling layer is dynamically stable. However, it is possible that the oil fouling accumulates overtime and even blocks the pipe, especially when the wax content in the oil is high and the adhered oil with wax builds up into hard structures. The mechanism of the 
stabilization of core-annular flow in a horizontal pipe is still an open question and discussions can be found in Ooms et al. ${ }^{9,31-33}$, Bannwart ${ }^{34}$, Rodriguez and Bannwart ${ }^{35}$, and Beerens et al. ${ }^{36}$

It is also noted that core annular flows with different characteristics are illustrated in Figure 3. More concentric CAF is reported for oils with higher densities and/or higher flow rates. Eccentric CAF is normally observed for oils with relatively lower densities and/or under higher water content. Besides, droplets may form at the interface of the phases.

\section{(Figure 3)}

From the above literature review, understanding on the flow patterns of liquid-liquid flow is summarized as follows. The diverse flow patterns in horizontal oil-water pipeline flow can be generally grouped into four basic categories: 1) stratified flow with either smooth or wavy interface (ST); 2) dispersed flow (D); 3) intermittent flow, including slugs/plugs and large drops of one phase in another phase (I); and 4) core annular flow (CAF). Other phase configurations can be regarded as transitional flow regimes between two of the above basic flow regimes. The oil viscosity plays an essential role in flow structures of oil-water flow, though the influence of oil viscosity may not be evident for certain ranges. Low-viscosity oil-water flow and highviscosity oil-water flow are characterized with different basic flow patterns. For low-viscosity oil-water flow, different flow characteristics are shown for density-matched oil-water flow, or for flow in small diameter pipes. This suggests that the density difference and pipe diameter also affect phase configurations. It is suspected that the quantitative change of flow system parameters, such as liquid viscosity, density and pipe diameter, leads to qualitative change of flow structures at critical conditions.

\section{Experimental Campaign and Results}

Though there is adequate experimental data on flow regimes of low-viscosity oil-water flow in the literature, experimental data on flow regimes of high-viscosity oil-water flow is quite limited due to the fact that many studies on high-viscosity oil-water flow focus on core annular flow thus other flow regimes are not covered or reported. An experimental campaign on highviscosity oil-water flow (two oils, oil viscosity range between 3300 and $16000 \mathrm{mPa} \cdot \mathrm{s}$ ) in a horizontal pipe (I.D.=26 mm) was conducted to collect experimental data on flow regimes. 
The experimental set-up is shown in Figure 4. The main part of the test rig where the flow is developed and the flow information is collected is a horizontal flow line with a length of 5.5 $\mathrm{m}$; this section is made from transparent Perspex for the purpose of visual observation. During tests, the flow was recorded using a digital HD video camera recorder (Sony HDR-CX550V). Detailed description of the experimental set-up and procedure is reported in Shi. ${ }^{21}$

\section{(Figure 4)}

Flow patterns' identification is mainly based on visual observation with pressure gradient values as reference. At a constant inlet oil velocity, with increase of the inlet water velocity (i.e., the input water volume fraction), the flow pattern evolves from the category of oilcontinuous flow to the category of water-continuous flow. Specific phase configurations of the oil-continuous flow were not visually observable in the experiments; they could be in forms of dispersion of large water drops or small droplets in oil. The different phase configurations of water-continuous flow can be divided into core annular flow, oil plugs in water and oil lumps in water as shown in Figure 5. In addition, transitional flow structures from oil-continuous to water-continuous flow characterized with dual continuous water and oil stream moving forward spirally or dual continuous water and oil spiral flow alternating with single oil flow were observed (see Figure 6); these phase configurations are named 'inversion' in this study. The inversion flow regime observed in the present study is an unstable transitional flow regime. It is unknown that whether stratified flow can be finally developed further downstream the mixing point in a longer pipe. The flow development length is not established for multiphase flow as it is different for different flow regimes and initial conditions. Whether this unstable inversion flow regime would stay the same transitional characteristics, become stable or even develop into stratified flow in a longer pipe is not investigated in the present study due to experimental limitation. It is noted that the other flow patterns as illustrated in Figure 5 are regarded as developed in the present study with regard to the stable pressure drop. In Figure 5, different degree of oil fouling ripples adjacent to the internal wall of the pipe can be clearly observed for the different water-lubricated flow regimes. As have been discussed above in the section Liquid-Liquid Flow Patterns in Horizontal Pipes - Literature Review, the existence of oil fouling 
can be related to the wall wettability of the pipe wall to the fluids and the experimental procedure. In this study, no special care was taken to avoid contact of oil to the pipe wall for the purpose of being more similar to industrial operations. More specifically, the fluids were mixed via a Tee junction; the pipe was not cleaned frequently with detergent to remove the oil fouling film on the pipe wall. To have an idea on the wall wettability to the fluids, a rough qualitative test was conducted by dropping small drops of oil and water via a syringe onto a Perspex plate and observing the contact angels between the solid wall and the liquids that are surrounded by air. It was showed that the Perspex material used in the experiments had a tendency to interact more with the oil used in the present study than with the water. However, it is noted that the contact angle observed in our simple test is different from that between the solid wall and a liquid phase that is surrounded by another liquid phase. A rigorous quantitative contact angle test is recommended for any future experimental investigation on oil-water twophase flow in this experimental facility.

\section{(Figure 5)}

\section{(Figure 6)}

Figure 7 descripts typical flow maps of oil-water flow based on our experimental results. The lines in Figure 7 are depicted for the purpose of distinguishing flow regimes; they represent approximate transition boundaries or, more strictly speaking, transition zones, between flow regimes. For the investigated oil viscosity range, the flow maps of oil-water flow are comparable. The major flow patterns observed are consistent and the transition trend between different flow patterns is also consistent. For the investigated oil viscosity range, the influence of oil viscosity on the inversion from oil-continuous to water-continuous flow appears minor. However, it can be observed that the region of CAF expands within the region of watercontinuous flow with increase of oil viscosity. The deformation and breakage of the oil core are associated with three types of forces - the external force from the annular water which gives rise to deformation, the interfacial tension which in general counteract the deformation, and viscous stresses caused by internal flows of the oil core owing to the deformation which also counteract the deformation. The internal viscous stress is usually small for low-viscosity 
oil compared to the interfacial tension, and the ratio of the external force to the interfacial tension, i.e., the Weber number (We), is normally used for the description of the deformation and breakage process of a dispersed phase. As discussed by Hinze ${ }^{41}$, when the viscous stresses become comparable to the interfacial tension, its role in counteracting the deformation cannot be ignored. Hinze ${ }^{41}$ used a dimensionless viscosity group $N_{v i}=\frac{\mu_{d}}{\sqrt{\rho_{d} \sigma D}}$, where $\sigma$ denotes the interfacial tension, $\mu_{d}$ the viscosity of the phase being deformed, and $\rho_{d}$ the density of the phase being deformed, to describe the influence of the viscosity of the phase being deformed and proposed that the critical Weber number for breakup increases with $N_{v i}$. For high-viscosity oil, the internal viscous stress together with the interfacial tension counteract the deformation preventing the oil core from breaking up. The internal viscous stresses is higher for a higher viscosity oil. This can explain why the transition from CAF to oil plugs or lumps is delayed for a higher oil viscosity in the flow map.

Comparisons of a flow pattern map produced from the present experimental campaign with selected literature data are presented in Figures 8 and 9. A summary of the experimental systems used for flow map comparison is descripted in Table 1. Due to small or large differences of the flow systems, inconsistency in the flow pattern maps is expected. The purpose of the comparison is to perceive the degree of variation of flow regime maps of different flow systems, hence the similarity of different liquid-liquid flows. Figure 8 shows a comparison of a flow pattern map produced from the present study with that produced by Sotgia et al. ${ }^{17}$ The core annular flow (CAF) defined in the present study corresponds to the CAF and wavy annular defined in Sotgia et al. ${ }^{17}$, the oil plugs corresponds to the slug flow, and the dispersed oil lumps to both the Transition and Dispersed flow. For the common region of interest, it can be observed that there is an overlapped area of CAF. For the whole region of each study, similar trends of transitions among flow regimes of oil plugs/slugs, CAF, and dispersed oil lumps are shown. The transition from oil plugs/slugs to annular flow occurs predominantly with increase of the superficial oil velocity, $U_{\text {so }}$; the transition from annular flow and oil plugs/slugs to dispersed oil lumps occurs predominantly with increase of the superficial 
water velocity, $U_{\text {sw. }}$ The major difference between the maps from the two studies is that wavy stratified flow covers the region of low $U_{\text {so }}$ and low $U_{\text {sw }}$ in Sotgia et al. ${ }^{17}$, while stratified flow was rarely observed in the present experiments. Instead, unstable inversion flow regime characterized with spiral motion of oil and water was observed at very low phase flow rates. Whether this unstable inversion flow regime in the present study would become stable or develop into stratified flow in a longer pipe is not known. As the pipe diameter and the interfacial tension are similar in the two studies, the oil viscosity and density are possible factors resulting in the difference. The gravitational force due to the density difference between oil and water which makes the phases stratified is less significant in the present study than that in Sotgia et $\mathrm{al}^{17}$. Also the higher oil viscosity in the present study favors the formation of CAF. Another factor which might cause the difference is the fluids injection sequence. In the present study, the oil was injected into the pipe followed by water from low to high flow rate to observe the inversion from oil-continuous flow to water-continuous flow. In the study of Sotgia et al., the opposite injection sequence was applied. ${ }^{17}$ It is shown in Figure 8 that the region of core annular flow extends as the oil viscosity increases. Also, for highly viscous oil, the stratified flow rarely develops or if it develops, its region is quite small comparing to core annular flow and dispersed oil lumps in water flow. The interface of stratified flow usually has a high curvature if stratified flow develops at limited conditions for high-viscosity oil-water flow. The wavy stratified flow with high interface curvature can be regarded as a transitional regime to the core annular flow. When the oil viscosity is very high, the gravitational force which tends to stratify two immiscible fluids must be competing with the interfacial tension and the internal viscous stress which keep the oil interface from breaking up and tend to minimize the shear between the pipe wall and the fluids.

In Figure 9, the flow maps from the present experiments and Trallero et al. ${ }^{1}$ are less comparable as the observed flow regimes are dramatically different. The only link is the occurrence of dispersed oil lumps in the present experiments and the dispersion of oil in water and water (Do/w \& w) in Trallero et al. ${ }^{1}$ at higher water volume fractions. The breakup of the oil phase occurs when the turbulent kinetic energy in the water is sufficiently high. For low 
viscosity oil, the oil phase is readily dispersed into droplets, while the oil is dispersed into lumps for highly viscous oil. The striking difference in the flow patterns is thought to come primarily from the large difference in oil viscosity. Most experimental studies on low-viscosity oil-water flow in the literature report flow regimes similar to those of Trallero et al. ${ }^{1}$ as has been introduced in the literature review section, while high-viscosity oil-water flow sees a major flow pattern of CAF.

\section{(Table 1)}

\section{(Figure 8)}

\section{(Figure 9)}

\section{Characterization of Liquid-Liquid Flows in Horizontal Pipes}

Upon the above sections, a question arises that under what conditions the liquid-liquid flows in horizontal pipes exhibit same basic flow regimes or under what conditions the flow regime maps of different liquid-liquid flows are comparable. Brauner ${ }^{37}$ proposed that a pipe flow system could be characterized by a dimensionless Eötvös $\left(\mathrm{Eo}^{\prime}\right)$ number. The Eo' is expressed as

$$
\mathrm{Eo}^{\prime}=\frac{\Delta \rho g D^{2}}{8 \sigma}
$$

where $\Delta \rho$ is the density differential, $\mathrm{kg} / \mathrm{m}^{3} ; g$ is the gravitational acceleration, $\mathrm{m} / \mathrm{s}^{2} ; \mathrm{D}$ is the pipe internal diameter, $\mathrm{m}$; and $\sigma$ is the interfacial tension, $\mathrm{N} / \mathrm{m}$. The factor ' 8 ' was introduced by the above author when the Young-Laplace equation for predicting the interface shape in stratified flow was solved, as this dimensionless group was evolved in the analysis in Brauner et al. ${ }^{42}$ More widely, the Eötvös number (Eo) is defined as

$$
\text { Eo }=\frac{\Delta \rho g D^{2}}{\sigma}
$$

In this paper, the notations of Eo' and Eo are used for specific and general context separately. The Eötvös number is the ratio of gravitational force to surface or interfacial tension. For liquidliquid flows with a small Eötvös number, the interfacial tension plays a dominant role and core annular flow is a natural configuration which complies with the interfacial tension. For liquid- 
liquid flows with a large Eötvös number, the gravitational force has more influence on the flow characteristics thus the flow tends to stratify. Brauner ${ }^{43}$ suggested that it is beneficial to preliminarily classify the liquid-liquid flow system according to whether $\mathrm{Eo}^{\prime}>>1$ or $\mathrm{Eo}^{\prime}<1$. This is a first attempt to characterize liquid-liquid systems in a big picture. Mechanisms for the formation of different flow patterns were discussed by the above author for flow systems of $\mathrm{Eo}^{\prime}>>1$ and $\mathrm{Eo}^{\prime}<1$ separately. However, as the effect of viscosity ratio is not included in $\mathrm{Eo}^{\prime}$, highly viscous oil-water flow systems were further dealt with separately. Also, there is ambiguity concerning the amplitude of the lower threshold of $\mathrm{Eo}^{\prime}$ for gravity dominated flow system $\left(\mathrm{Eo}^{\prime}>>1\right)$. A criterion for the existence of core annular flow was proposed by Bannwart ${ }^{44}$ making use of $\mathrm{Eo}^{\prime}$. The criterion is that CAF is likely to form when $\mathrm{Eo}^{\prime}<\frac{4}{\pi \varepsilon}(\varepsilon$ is the volume fraction of the core, $\mathrm{Eo}^{\prime}<2.55$ with $\left.\varepsilon=0.5\right)$.

A survey on the Eo' of different liquid-liquid flows was conducted as included in Table 2. We can observe that CAF tends to form in systems with low $\mathrm{Eo}^{\prime}$, while CAF can also be formed in systems with higher $\mathrm{Eo}^{\prime}$, e.g., experiments of Sridhar et al. ${ }^{18}$, McKibben et al. ${ }^{14}$ and Ooms et al. ${ }^{9}$ It is noted that when CAF is formed in flow systems with higher $\mathrm{Eo}^{\prime}$, the systems are characterized with high-viscosity oil.

The Eötvös number incorporates the parameters of phase densities, pipe diameter, and surface tension which can affect flow structures of oil-water flow. However, the oil viscosity is not included in this dimensionless number. From the above review on flow patterns of liquidliquid flows in horizontal pipes, it is demonstrated that the oil viscosity plays an essential role in phase configuration of oil-water flows. The influence of interfacial tension on phase configuration is not clearly reflected in Table 2 . The interfacial tension between oil and water varies from 0.017 to $0.062 \mathrm{~N} / \mathrm{m}$ in the survey and CAF can be formed under either a high or low interfacial tension.

To include the role that oil viscosity plays in the phase configuration of liquid-liquid flows, it is proposed to use the ratio of the gravitational force to viscous force to characterize liquid- 
liquid flows featured with different basic flow regimes. The gravitation to viscous force ratio can be expressed as

$$
\mathrm{G} / \mathrm{V}=\frac{\Delta \rho g D^{2}}{\mu U}
$$

The gravitation to viscous force ratio reflects the competitive role of gravitational force and viscous force on phase configuration of oil-water flows. The viscous force is expressed as the product of the characteristic viscosity, $\mu$, and velocity, $U$; the viscosity and velocity of the more viscous phase, normally the oil phase, i.e., $\mu_{o}$ and $U_{o}$ are used as the characteristic viscosity and velocity. When the local oil phase velocity is not known, the mixture velocity, $U_{m}$, can be used as the characteristic velocity. For liquid-liquid flows with a high $\mathrm{G} / \mathrm{V}$, the gravitational force dominates, and the viscous force is relatively small hence the fluid can be broken up readily when the flow kinetic energy is high, stratified flow (ST) or fine dispersions of one fluid in another (D) are two basic flow structures developed. For liquid-liquid flows with a low $G / V$, the effect of the gravitational force is relatively small hence stratified flow is less likely to form. The flow configures itself in forms of oil core flowing inside annular water, continuously to form annular (CAF) or discontinuously to form intermittent (I), in which the shear stress can be minimized. Also when the flow turbulence kinetic energy is high, the higher viscosity phase is too viscous to be broken into fine drops but lumps of irregular sizes and shapes. This kind of flow structure can be regarded as a transition from intermittent to the ultimate dispersed flow (I-D). For liquid-liquid flows with a medium $\mathrm{G} / \mathrm{V}$, the gravitational force and the viscous force together affect flow structures and all the four basic phase configurations, i.e., ST, CAF, I, D, can be developed.

It is noted that the gravitation to viscous force ratio can be expressed as the ratio of the Eötvös number (Eo) to the Capillary number (Ca), or the ratio of the Reynolds number $(\mathrm{Re})$ to the Froude number $(\mathrm{Fr})$, i.e., $\mathrm{G} / \mathrm{V}=\mathrm{Eo} / \mathrm{Ca}=\mathrm{Re} / \mathrm{Fr}$. The Eötvös number represents the relative effect of the gravitational force versus interfacial tension as introduced in Equation (2). The capillary number represents the relative effect of the viscous force versus interfacial tension between two immiscible liquids (see Equation (4)). The Reynolds number represents 
the relative effect of the inertial force versus viscous force, and the Froude number the relative effect of the inertial force versus gravitational force (see Equations (5) and (6)).

$$
\begin{gathered}
\mathrm{Ca}=\frac{\mu U}{\sigma} \\
\operatorname{Re}=\frac{\rho U D}{\mu} \\
\mathrm{Fr}=\frac{U}{\sqrt{g D \frac{\Delta \rho}{\rho}}}=\frac{U^{2}}{g D \frac{\Delta \rho}{\rho}}
\end{gathered}
$$

For the gravitation to viscous force ratio, the interfacial tension and inertial force are not included. The interfacial tension and inertial force are also important in the phase configuration of liquid-liquid flow. However, when looking at liquid-liquid flows characterized with different basic flow regimes instead of specific flow regimes, it is thought that the gravitational force and viscous force are the most influential forces.

A summary of liquid-liquid flows in horizontal pipes with attention on the basic flow patterns developed and the corresponding G/V are presented in Table 2. As the oil phase average velocity is usually not known, the mixture flow velocity is used as the characteristic velocity to estimate the gravitation to viscous force ratio. It can be observed that the magnitude of this dimensionless number corresponds to certain flow patterns as analyzed above. For liquidliquid flows with high gravitation to viscous force ratio, only ST and D are formed, for example, studies of Trallero et al. ${ }^{1}$, Vielma et al. ${ }^{2}$, Nädler and Mewes ${ }^{3}$, Angeli and Hewitt ${ }^{4}$ and Lovick and Angeli ${ }^{5}$. For liquid-liquid flows with low gravitation to viscous force ratio, CAF and I are the basic flow regimes, for example, studies of Arney et al. ${ }^{12}$, McKibben et al. ${ }^{14}$, Al-Awadi ${ }^{19}$, Alagbe ${ }^{20}$ and $\mathrm{Shi}^{21}$. It is noted that one exception is the study of Charles et al. ${ }^{6}$ of which the gravitational force or buoyancy force to stratify the fluids is not affecting the phase configuration. This study is excluded in this context in our analysis due to the essential difference for density-matched liquid-liquid flow. Some studies have a gravitation to viscous force ratio range overlapping both the high and the medium categories, e.g., Russell et al. ${ }^{7}$ and Sridhar et al. ${ }^{18}$, thus the reported flow patterns are among the possible flow patterns in 
both gravitational force dominant flow and gravitational force and viscous force comparable flow. Also, some studies have low to medium gravitation to viscous force ratios, e.g., Grassi et al. ${ }^{16}$ and Sotgia et al. ${ }^{17}$, thus the reported flow patterns are among the possible flow patterns in both viscous force dominant flow and gravitational force and viscous force comparable flow.

The change of basic flow patterns of liquid-liquid flows with the gravitation to viscous force ratio, $G / V=\frac{\Delta \rho g D^{2}}{\mu U}$, is illustrated by the diagram shown in Figure 10. An oil-water flow system can be gravitational force dominant flow $\left(\mathrm{S}_{\mathrm{G}}\right)$, viscous force dominant flow $\left(\mathrm{S}_{\mathrm{v}}\right)$, or gravitational force and viscous force comparable flow $\left(\mathrm{S}_{\mathrm{GV}}\right)$. One can expect that the interface of stratified flow in the category of $S_{G V}$ has a higher curvature under effects of both the gravitational force and viscous force, which is different from the relatively flatter phase interface of stratified flow in the category of $\mathrm{S}_{\mathrm{G}}$. Also, the dispersed flow in the category of $\mathrm{S}_{\mathrm{GV}}$ is different from the dispersed flow in the category of $S_{G}$ in terms of drop sizes and/or shapes.

\section{(Figure 10)}

The different flow maps produced from different experiments would be more comparable when the liquid-liquid flow systems are dominated by same force(s). Figures 11 to 15 demonstrate comparisons of flow maps with coordinates of the gravitation to viscous force ratio $(\mathrm{G} / \mathrm{V})$ and input water volume fraction $\left(C_{\mathrm{w}}\right)$. They are re-presentations of reported flow maps in the literature with either the superficial phase velocities $\left(U_{\mathrm{so}}\right.$ and $\left.U_{\mathrm{sw}}\right)$ or the input water volume fraction and the mixture velocity $\left(C_{\mathrm{w}}\right.$ and $\left.U_{\mathrm{m}}\right)$ as coordinates.

Figure 11 shows a flow map for the gravitational force dominant oil-water flows $\left(\mathrm{S}_{\mathrm{G}}\right)$. It is adapted from five different flow maps produced by Trallero et al. ${ }^{1}$, Vielma et al. ${ }^{2}$, Nädler and Mewes $^{3}$, Angeli and Hewitt ${ }^{4}$ and Lovick and Angeli ${ }^{5}$. The five flow systems have diameters ranging from 24 to $59 \mathrm{~mm}$, oil densities from 801 to $884 \mathrm{~kg} / \mathrm{m}^{3}$, and oil viscosity from 1.6 to 28.8 $\mathrm{mPa}$.s. The basic flow patterns reported for these flow systems are ST and D. These oilwater flows locate in a range of G/V from 40 to 4000 approximately. It is interesting to observe that the stratified flow covers the range of $G / V$ from 400 to 4000 approximately, and the dispersed flow covers the range of G/V from 40 to 400 approximately. It should be noted that 
the transitional region between the ST and D varies for different flow systems. The dimensionless gravitation to viscous force ratio is proposed to distinguish liquid-liquid flow systems instead of specific flow regimes.

\section{(Figure 11)}

\section{(Figure 12)}

Specific flow regimes for the dispersed flow are shown in Figure 12. It demonstrates that the dispersed water in oil (Dw/o) develops at low $C_{\mathrm{w}}$, and the dispersed oil in water (Do/w) develops at high $C_{w}$. The dual-continuous flow such as Dw/o \& W and Dw/o \& Do/w develops at medium $C_{\mathrm{w}}$ around 0.4 ; this is a transitional buffer zone from oil-continuous to watercontinuous flow. In general, the transition from oil-continuous flow to dual-continuous occurs at a lower $C_{w}$ with decrease of $G / V$. The decrease of $G / V$ is mainly caused by increase of the oil viscosity or mixture velocity. To maintain a stable Dw/o, the turbulence level in the continuous oil phase needs to be sufficiently high to disperse the water phase into stable small droplets. The turbulent kinetic energy of the oil phase decreases with increase of viscosity. Though the turbulent kinetic energy of the oil phase increases with increase of the mixture velocity, the turbulent kinetic energy of the water phase increases at the same time and can increase more quickly with increase of the mixture velocity due to a lower viscosity. When the turbulence level in the water phase is sufficiently high, the water phase can disperse the oil phase into drops initiating the transition from oil-continuous to dual-continuous flow. The transition from dual-continuous flow to water-continuous or the completion of the inversion occurs at a higher $C_{w}$ for a lower $G / V$ caused by the increase of the oil viscosity when comparing different experimental studies. This can be explained similarly from the degree of the phase turbulent kinetic energy. To maintain a stable Do/w, the turbulence level in the continuous water phase needs to be sufficiently high to disperse the oil phase into stable small droplets. When the oil is more viscous, the water phase needs higher turbulent kinetic energy to disperse the oil phase thus the critical water volume fraction to form stable Do/w increases. It is worth remarking that apart from the influences of oil viscosity and mixture velocity, other parameters such as the interfacial tension, phase volume fraction increase method 
(continuously or discontinuously) and transition direction (from oil-continuous to watercontinuous or the opposite) can also affect the transition from Dw/o to Do/w. ${ }^{24-28,37}$ It is hardly possible to include all the influences of different parameters on the inversion in one map.

Figure 13 shows a flow map for gravitational force and viscous force comparable flow ( $\left.\mathrm{S}_{\mathrm{GV}}\right)$. This flow map is adapted from the two flow maps produced by Wegmann and Rudolf von Rohr $^{10}$. The above authors conducted oil-water experiments in two small diameter pipes (5.6 and $7 \mathrm{~mm}$ ) with a low-viscosity oil (4.3-5.2 $\mathrm{mPa} \cdot \mathrm{s})$. All the four basic flow patterns, i.e., ST, CAF, I, and D, are reported for these flow systems. The flow systems cover the range of G/V between 2 and 40 . The stratified flow mainly covers the region of low to medium $C_{\mathrm{w}}$ and $\mathrm{G} / \mathrm{V}$ between 10 and 40 . The core annular flow mainly covers the region of low to medium $C_{w}$ and G/V between 2 and 10. The intermittent flow develops at medium to high $\mathrm{Cw}$ and $\mathrm{G} / \mathrm{V}$ between 2 and 40.

\section{(Figure 13)}

\section{(Figure 14)}

Figure 14 shows flow maps produced by Grassi et al. ${ }^{16}$, Sotgia et al. ${ }^{17}$ and the present study. The liquid-liquid flow investigated in the present study is mainly viscous force dominant flow having G/V lower than 1. The liquid-liquid flows investigated by Grassi et al. ${ }^{16}$ and Sotgia et al. ${ }^{17}$ cover low to medium G/V. It shows that the transition from gravitational force and viscous force comparable flow to viscous force dominant flow is around $\mathrm{G} / \mathrm{V}=1$. Below $1, \mathrm{ST}$ is rarely formed; CAF and I are the two basic flow patterns. The CAF covers a wide range of $C_{\mathrm{w}}$. At very low $C_{\mathrm{w}}$, the flow is oil-continous flow, and at very high $C_{\mathrm{w}}$, the flow is intermittent flow (I) or transition from intermittent flow to disperased flow (I-D). It is noted that the term of dispersed flow was used by Grassi et al. ${ }^{16}$ and Sotgia et al. ${ }^{17}$ to describe dispersed oil lumps in water. In this context, the dispersed flow defined by the above authors is grouped into I \& I-D.

Combing all the experimental test points from Figures 11 to 14 , a flow map is shown in Figure 15 with $S_{G}, S_{G V}$, and $S_{V}$ covering different areas. The capability of the proposed gravitation to viscous force ratio, G/V, to categorize different liquid-liquid flows is demonstrated. At low $G / V(G / V<1)$, the liquid-liquid flow is viscous force dominant flow. The liquid-liquid flow 
becomes gravitational force and viscous force comparable flow when $G / V$ increases to above a critical value around 1. The gravitational force and viscous force comparable flow becomes gravitational force dominant flow when $\mathrm{G} / \mathrm{V}$ further increases to above a critical value around 40. These transitions of liquid-liquid two-phase flows can be analogical to the laminar-toturbulent transition in single phase flow. Similarly, the transitions between the different categories of liquid-liquids flows are buffer zones. Other parameters such as the pipe wall roughness, interfacial tension and initial conditions can affect the exact transition thresholds of $\mathrm{G} / \mathrm{V}$.

\section{(Figure 15)}

The proposed characterization of liquid-liquid flows in horizontal pipes with the dimensionless gravitation to viscous force ratio sheds some light on the physics underlying the liquid-liquid flows with different basic flow patterns. Since liquid-liquid flows can be viscous force dominated $\left(\mathrm{S}_{\mathrm{V}}\right)$, gravitational force dominated $\left(\mathrm{S}_{\mathrm{G}}\right)$, or both viscous force and gravitational force affected $\left(\mathrm{S}_{\mathrm{GV}}\right)$, different basic phase configurations can be developed. This explains the fact that the flow regime maps are consistent for some systems of liquid-liquid flow, while not for some other systems of liquid-liquid flow. Also, the approximate thresholds of $\mathrm{G} / \mathrm{V}$ for the transitions of different flow categories is of particle use to help judge the possible basic phase configurations.

\section{Conclusions}

A literature review on flow patterns of liquid-liquid two-phase flow in horizontal pipes was conducted. The ratio of the gravitational force to viscous force $\left(\mathrm{G} / \mathrm{V}=\frac{\Delta \rho g D^{2}}{\mu U}\right)$ was proposed and validated to characterize liquid-liquid two-phase flows featuring different basic flow regimes. Liquid-liquid flows with basic flow patterns of ST and D are gravitational force dominant and characterized with high G/V. Liquid-liquid flows with basic flow patterns of CAF and I are viscous force dominant and characterized with low G/V. Liquid-liquid flows with basic flow patterns of ST, D, CAF and I are gravitational force and viscous force comparable and characterized with medium G/V. The transitions of two-phase liquid-liquid flows can be roughly 
described with the gravity-viscosity ratio. The transition from viscous force dominant flow to gravitational force and viscous force comparable flow is around a gravitation to viscous force ratio of 1 . The transition from gravitational force and viscous force comparable flow to gravitational force dominant flow is around a gravitation to viscous force ratio of 40 . Parameters such as the pipe wall roughness, interfacial tension and initial conditions can affect the exact transition thresholds of G/V. This characterization of liquid-liquid flows in horizontal pipes with the dimensionless gravitation to viscous force ratio sheds some light on the physics underlying the liquid-liquid flows with different basic flow patterns. Mechanisms for the formation of different specific flow patterns can be further studied upon the above classification of liquid-liquid flows.

\section{Literature Cited}

1. Trallero JL, Sarica C, Brill JP. A Study of Oil-Water Flow Patterns in Horizontal Pipes. SPE Prod. \& Facil. 1997;12(03):165-172.

2. Vielma M, Atmaca S, Sarica C, Zhang H-Q. Characterization of oil/water flows in horizontal pipes. SPE Proj Fac \& Const. 2008;3(04):1-21.

3. Nädler M, Mewes D. Flow induced emulsification in the flow of two immiscible liquids in horizontal pipes. Int J Multiphase Flow. 1997;23(1):55-68.

4. Angeli P, Hewitt GF. Flow structure in horizontal oil-water flow. Int J Multiphase Flow. 2000;26(7):1117-1140.

5. Lovick J, Angeli P. Experimental studies on the dual continuous flow pattern in oil-water flows. Int J Multiphase Flow. 2004;30(2):139-157.

6. Charles ME, Govier GW, Hodgson GW. The horizontal pipeline flow of equal density oilwater mixtures. Can. J. Chem. Eng. 1961;39(1):27-36.

7. Russell TWF, Hodgson GW, Govier GW. Horizontal pipeline flow of mixtures of oil and water. Can. J. Chem. Eng. 1959;37:9-17.

8. Wegmann A, Rudolf von Rohr P. Two phase liquid-liquid flows in pipes of small diameters. Int J Multiphase Flow. 2006;32(8):1017-1028. 
9. Ooms G, Segal A, van der Wees AJ, Meerhoff R, Oliemans RVA. A theoretical model for core-annular flow of a very viscous oil core and a water annulus through a horizontal pipe. Int J Multiphase Flow. 1984;10(1):41-60.

10. Oliemans RVA, Ooms G, Wu HL, Duijvestijn A. Core-annular oil/water flow: the turbulentlubricating-film model and measurements in a $5 \mathrm{~cm}$ pipe loop. Int $J$ Multiphase Flow. $1987 ; 13(1): 23-31$.

11. Bannwart AC. Wavespeed and volumetric fraction in core annular flow. Int $J$ Multiphase Flow. 1998;24(6):961-974.

12. Arney MS, Bai R, Guevara E, Joseph D, Liu K. Friction factor and holdup studies for lubricated pipelining-I. Experiments and correlations. Int J Multiphase Flow. 1993;19(6), 1061-1076.

13. McKibben MJ, Gillies RG, Shook CA. A laboratory investigation of horizontal well heavy oil-water flows. Can. J. Chem. Eng. 2000;78(4):743-751.

14. McKibben MJ, Gillies RG, Shook CA. Predicting pressure gradients in heavy oil-water pipelines. Can. J. Chem. Eng. 2000;78(4):752-756.

15. Bannwart AC, Rodriguez OM, de Carvalho $\mathrm{CH}$, Wang IS, Vara RM. Flow patterns in heavy crude oil-water flow. J. Energy Resour. Technol. 2004;126(3):184-189.

16. Grassi B, Strazza D, Poesio P. Experimental validation of theoretical models in two-phase high-viscosity ratio liquid-liquid flows in horizontal and slightly inclined pipes. Int $J$ Multiphase Flow. 2008;34(10):950-965.

17. Sotgia G, Tartarini P, Stalio E. Experimental analysis of flow regimes and pressure drop reduction in oil-water mixtures. Int J Multiphase Flow. 2008; 34(12): 1161-1174.

18. Sridhar S, Zhang H-Q, Sarica C, Pereyra E. Experiments and Model Assessment on HighViscosity Oil/Water Inclined Pipe Flows. In: SPE Annual Technical Conference and Exhibition, SPE 146448, Denver, Colorado, 2011.

19. Al-Awadi H. Multiphase characteristics of high viscosity oil. PhD Thesis, Cranfield:Cranfield University; 2011. 
20. Alagbe SO. Experimental and numerical investigation of high viscosity oil-based multiphase flows. PhD Thesis, Cranfield:Cranfield University; 2013.

21. Shi J. A study on high-viscosity oil-water two-phase flow in horizontal pipes. PhD Thesis, Cranfield:Cranfield University; 2015.

22. Rodriguez $\mathrm{OMH}$, Baldani LS. Prediction of pressure gradient and holdup in wavy stratified liquid-liquid inclined pipe flow. J. Pet. Sci. Eng. 2012;96:140-151.

23. Rodriguez $\mathrm{OMH}$, Castro MS. Interfacial-tension-force model for the wavy-stratified liquidliquid flow pattern transition. Int J Multiphase Flow. 2014;58:114-126.

24. Ioannou K, Nydal OJ, Angeli P. Phase inversion in dispersed liquid-liquid flows. Exp. Therm. Fluid Sci. 2005;29(3):331-339.

25. Piela K, Delfos R, Ooms G, Westerweel J, Oliemans R, Mudde R. Experimental investigation of phase inversion in an oil-water flow through a horizontal pipe loop. Int $J$ Multiphase Flow. 2006;32(9):1087-1099.

26. Piela K, Delfos R, Ooms G, Westerweel J, Oliemans R. On the phase inversion process in an oil-water pipe flow. Int J Multiphase Flow. 2008;34(7):665-677.

27. Ngan KH, loannou K, Rhyne LD, Wang W, Angeli P. A methodology for predicting phase inversion during liquid-liquid dispersed pipeline flow. Chem. Eng. Res. Des. 2009;87(3):318324.

28. Piela K, Delfos R, Ooms G, Westerweel J, Oliemans R. Dispersed oil-water-gas flow through a horizontal pipe. AIChE J. 2009;55(5):1090-1102.

29. Oliemans RVA, Ooms G. Core-annular flow of oil and water. Multiphase Sci. Technol. $1986 ; 2(1-4): 427-476$.

30. Joseph DD, Bai R, Chen K, Renardy YY. Core-annular flows. Annu. Rev. Fluid Mech.1997;29(1):65-90.

31. Ooms G, Poesio P. Stationary core-annular flow through a horizontal pipe. Phys. Rev. E. 2003;68(6):066301 .

32. Ooms G, Vuik C and Poesio P. Core-annular flow through a horizontal pipe: hydrodynamic counterbalancing of buoyancy force on core. Phys. Fluids (1994-present). 2007;19(9):092103. 
33. Ooms G, Pourquie MJ and Beerens JC. On the levitation force in horizontal core-annular flow with a large viscosity ratio and small density ratio. Phys. Fluids (1994-present). 2013; 25(3):032102.

34. Bannwart AC. Bubble analogy and stabilization of core-annular flow. J. Energy Resour. Technol. 2001;123(2):127-132.

35. Rodriguez OM, Bannwart AC. Stability analysis of core-annular flow and neutral stability wave number. AIChE J. 2008;54(1):20-31.

36. Beerens JC, Ooms G, Pourquie MJ, Westerweel J. A comparison between numerical predictions and theoretical and experimental results for laminar core-annular flow. AlChE J. 2014;60(8), pp.3046-3056.

37. Brauner N. Liquid-Liquid Two-Phase Flow. In Hewitt GF(Ed.), The Update J. of the Heat Exchanger Design Handbook. New York:Begell House, 1998.

38. Barnea D, Luninski Y, Taitel Y. Flow pattern in horizontal and vertical two phase flow in small diameter pipes. Can. J. Chem. Eng. 1983;61(5):617-620.

39. Beretta A, Ferrari P, Galbiati L, Andreini P. Horizontal oil-water flow in small diameter tubes. Flow patterns. Int. Commun. Heat Mass Transfer. 1997;24(2): 223-229.

40. Tang X, Richter S, Nesic S. Study of wettability of different mild steel surfaces. In: Int. Corros. Congr., 17th. Las Vegas: NACE International; 2008:1298-1315.

41. Hinze JO. Fundamentals of the hydrodynamic mechanism of splitting in dispersion processes. AIChE J. 1955;1(3):289-295.

42. Brauner N, Moalem Maron D, Rovinsky J. A two-fluid model for stratified flows with curved interfaces. Int J Multiphase Flow.1998;24(6):975-1004.

43. Brauner N. Liquid-Liquid Two-Phase Flow Systems. In Bertola V(Ed.), Modeling and Control of Two-Phase Flow Phenomena. Italy:CISM Center, 2002.

44. Bannwart AC. Modeling aspects of oil-water core-annular flows. J. Pet. Sci. Eng. $2001 ; 32(2): 127-143$. 


\section{Figure and Table Captions}

Figure 1. Sketches of flow patterns of low-viscosity, unequal-density oil-water flow in horizontal pipes (adapted from Trallero et al. ${ }^{1}$ and Nädler and Mewes ${ }^{3}$ ).

Figure 2. Sketches of flow patterns of low-viscosity, equal-density oil-water flow in horizontal pipes (adapted from Charles et al. ${ }^{6}$ ).

Figure 3. Sketches of flow patterns of water-continuous high-viscosity oil-water flow in horizontal pipes.

Figure 4. Schematic diagram of experimental setup.

Figure 5. Pictures of typical flow regimes in experiments. a- Oil-continuous; b1, b2-Core annular flow; c-Oil plugs in water; d1, d2-Oil lumps in water.

Figure 6. Pictures of the inversion flow regime.

Figure 7. Experimental flow pattern maps of oil-water flow in a 1-inch pipe for oil viscosity 3300 and $7100 \mathrm{mPa} \cdot \mathrm{s}$.

Figure 8. Comparison of flow pattern maps from the present study and Sotgia et al. ${ }^{17}$ Regimes defined in the present study are presented with markers. Regimes defined by Sotgia et al. ${ }^{17}$ are presented with boundary lines and regime descriptions: Wavy stratified; Wavy annular; CAF; Slug; Transition (between CAF/Slug and Dispersed); Dispersed.

Figure 9. Comparison of flow pattern maps from the present study and Trallero et al. ${ }^{1}$ Regimes defined in the present study are presented with markers. Regimes defined by Trallero et al. ${ }^{1}$ are presented with boundary lines and regime descriptions: Stratified (ST); Stratified with mixing at the interface (ST \& MI); Dispersion of oil in water and water (Do/w \& w); Oil in water emulsion (o/w); Dispersion of water in oil and oil in water (Dw/o \& Do/w); Water in oil emulsion (w/o).

Figure 10. A diagram describing liquid-liquid flows characterized with different basic flow patterns and the corresponding gravitation to viscous force ratio (G/V). Figure 11. Basic flow patterns developed with gravitation to viscous force ratio (G/V) and $\mathrm{C}_{\mathrm{w}}$ in gravitational force dominant oil-water flows $\left(\mathrm{S}_{\mathrm{G}}\right)$. 
Figure 12. Specific dispersed flow patterns with gravitation to viscous force ratio $(\mathrm{G} / \mathrm{V})$ and $\mathrm{C}_{\mathrm{w} \cdot}$

Figure 13. Basic flow patterns developed with gravitation to viscous force ratio (G/V) and $\mathrm{C}_{\mathrm{w}}$ in gravitational force and viscous force comparable oil-water flows ( $\left.\mathrm{S}_{\mathrm{GV}}\right)$. Figure 14. Basic flow patterns developed with gravitation to viscous force ratio (G/V) and $\mathrm{C}_{\mathrm{w}}$ in mainly viscous force dominant oil-water flows $\left(\mathrm{S}_{\mathrm{v}}\right)$.

Figure 15. Basic flow patterns developed with gravitation to viscous force ratio (G/V) and $\mathbf{C}_{\mathrm{w}}$ in two-phase oil-water flows, $\mathbf{S}_{\mathrm{G}}, \mathbf{S}_{\mathrm{v}}$ and $\mathbf{S}_{\mathrm{Gv}}$. Source data: Trallero et al. ${ }^{1}$, Vielma et al. ${ }^{2}$, Nädler and Mewes ${ }^{3}$, Angeli and Hewitt ${ }^{4}$, Lovick and Angeli ${ }^{5}$, Wegmann and Rudolf von Rohr ${ }^{8}$, Grassi et al. ${ }^{16}$, Sotgia et al. ${ }^{17}$ and present study. The bold lines represents approximate transitions between $\mathrm{S}_{\mathrm{G}}, \mathrm{S}_{\mathrm{GV}}$, and $\mathrm{S}_{\mathrm{V}}$.

Table 1 Summary of the data sets used for flow map comparison.

Table 2 Summary of experimental studies on liquid-liquid flows in horizontal pipes. 


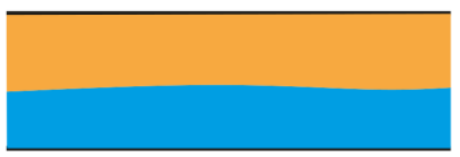

Stratified flow (ST)
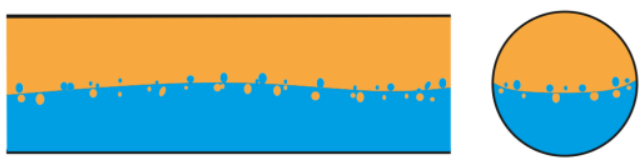

Stratified flow with mixing at the interface (ST \& MI)
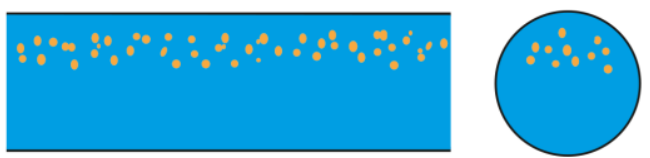

Dispersion of oil in water and water (Do/w \& w)
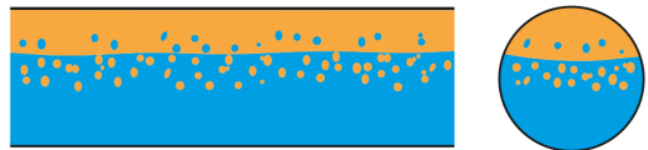

Dispersion of water in oil and oil in water and water (Dw/o \& o/w \& w)
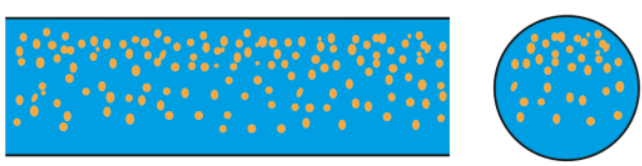

Oil in water emulsion (Do/w)
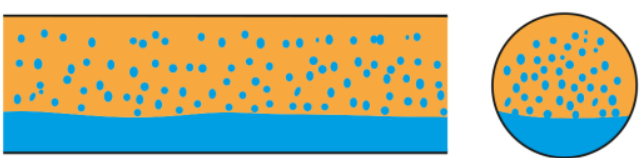

Dispersion of water in oil and water (Dw/o \& w)
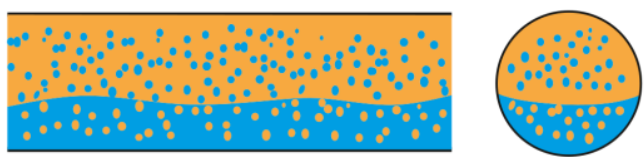

Dispersion of water in oil and oil in water (Dw/o \& o/w)
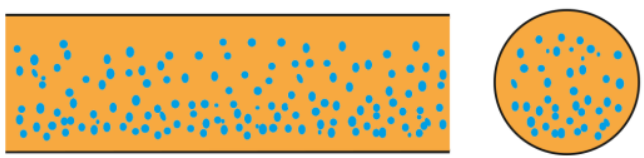

Figure 1. Sketches of flow patterns of low-viscosity, unequal-density oil-water flow in horizontal pipes (adapted from Trallero et al. ${ }^{1}$ and Nädler and Mewes ${ }^{3}$ ). 


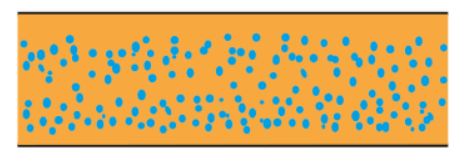

Water in oil emulsion (Dw/o)

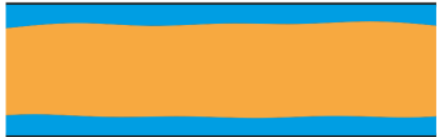

Core annular flow (CAF)

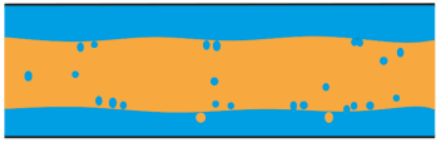

Core annular flow (CAF)
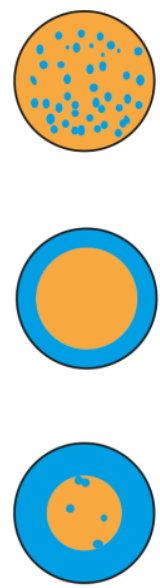

Oil slugs/plugs in water (OPL)

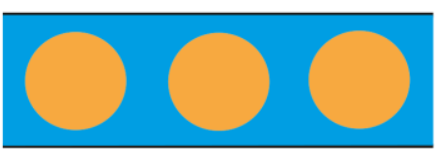

Large oil drops in water (ODR)

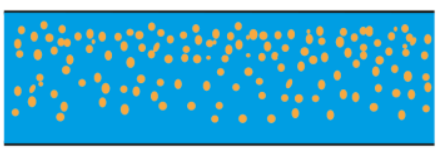

Oil in water emulsion (Do/w)
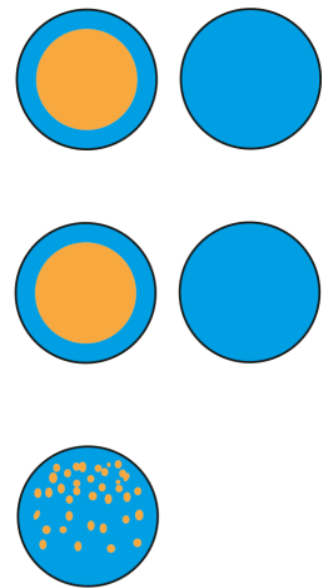

Figure 2. Sketches of flow patterns of low-viscosity, equal-density oil-water flow in horizontal pipes (adapted from Charles et al. ${ }^{6}$ ). 


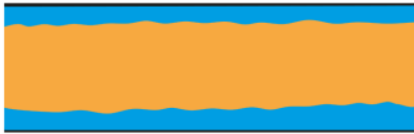

Core annular flow (CAF)

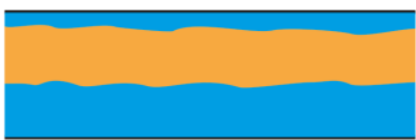

Core annular flow (CAF)

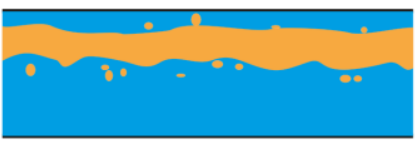

Core annular flow (CAF)

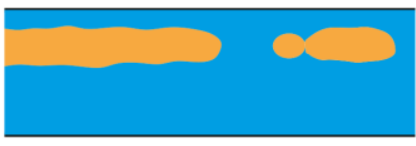

Oil plugs in water (OPL)
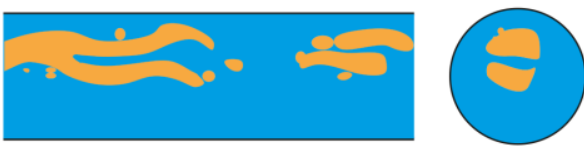

Dispersed oil lumps in water (OLP)
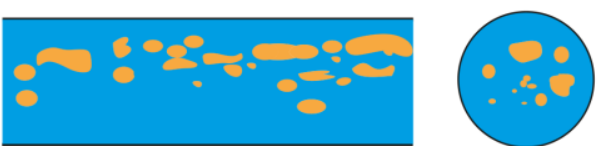

Dispersed oil lumps in water (OLP)
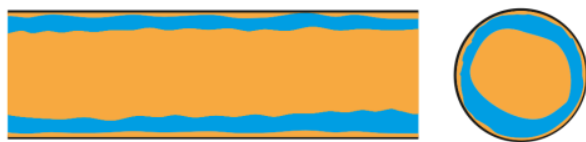

Core annular flow (CAF)
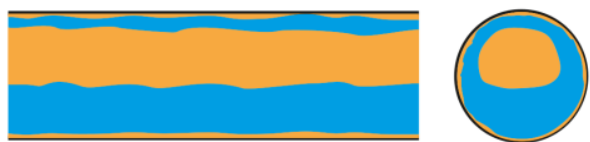

Core annular flow (CAF)
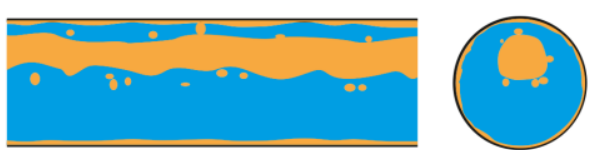

Core annular flow (CAF)
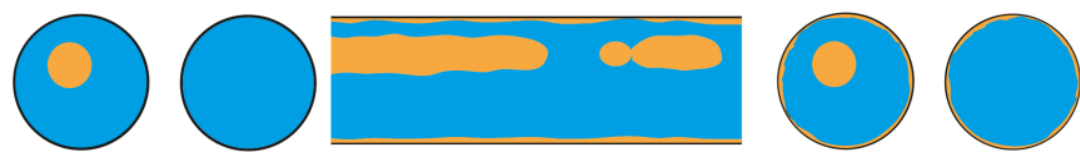

Oil plugs in water $(\mathrm{OPL})$
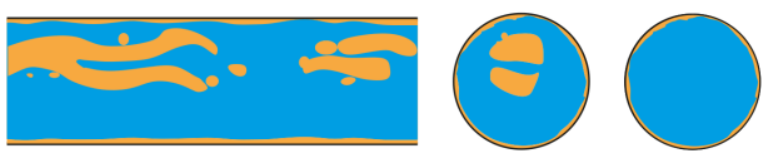

Dispersed oil lumps in water (OLP)
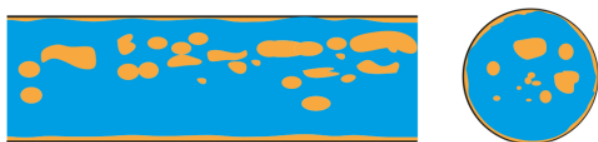

Dispersed oil lumps in water (OLP)

Figure 3. Sketches of flow patterns of water-continuous high-viscosity oil-water flow in horizontal pipes. 


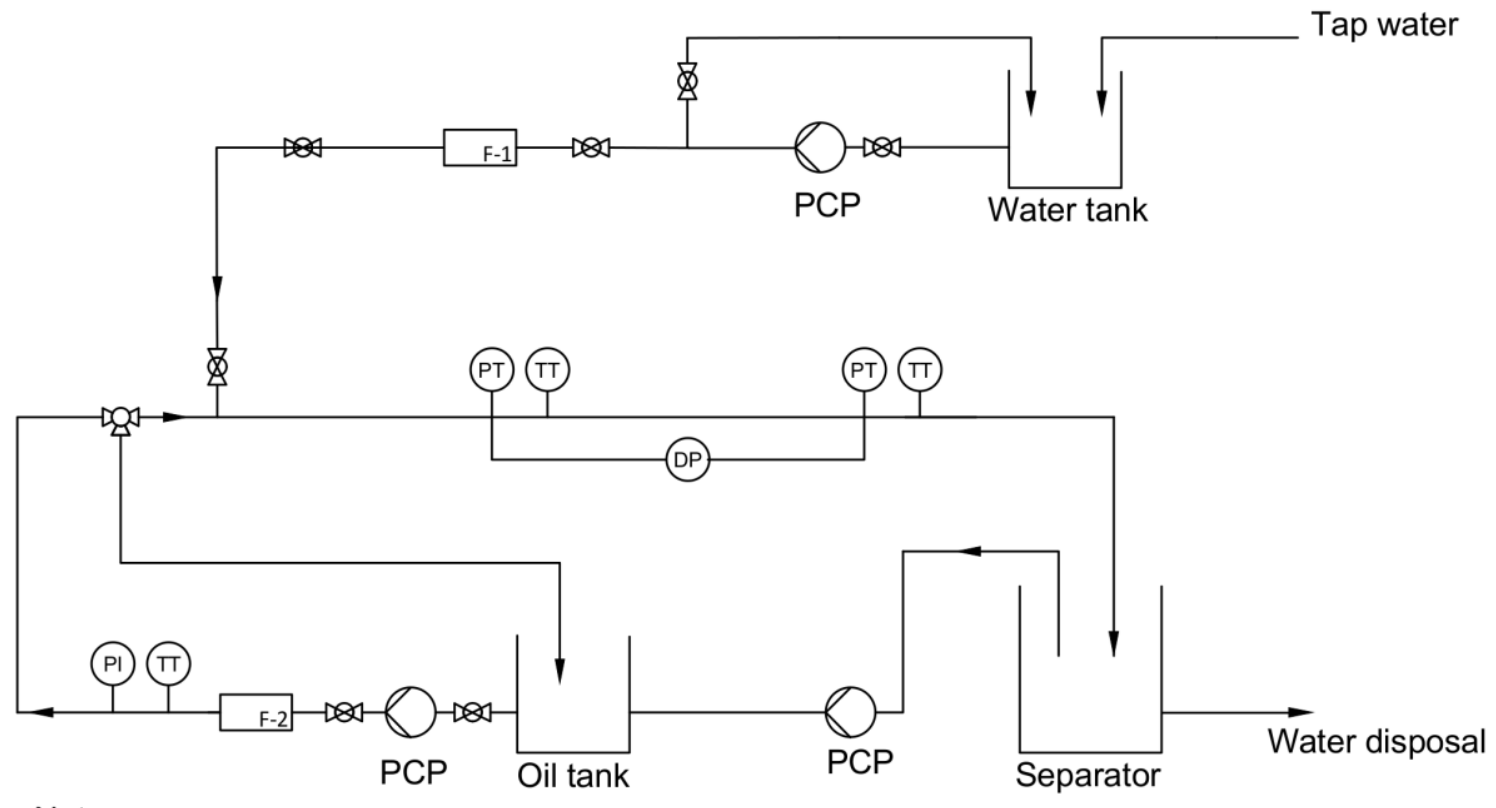

Notes:

PCP: Progressive cavity pump; F-1: Electromagnetic flowmeter;

PT: Pressure transducer;

DP: differential pressure transducer;

F-2: Coriolis flowmeter

TT: Temperature transducer

$\mathrm{PI}$ : Pressure indicator

Figure 4. Schematic diagram of experimental setup. 
(a)

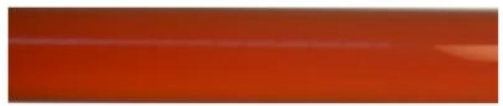

(b1)

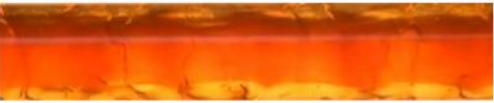

(b2)

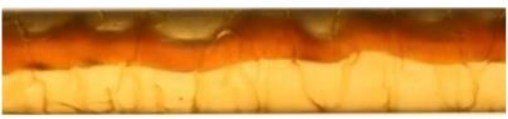

(c)

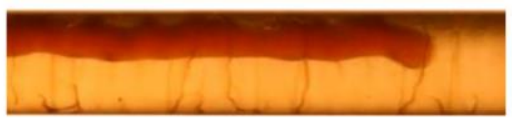

(d1)

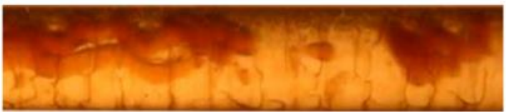

(d2)

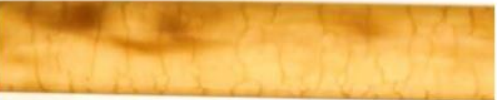

Figure 5. Pictures of typical flow regimes in experiments. a- Oil-continuous; b1, b2-Core annular flow; c-Oil plugs in water; d1, d2-Oil lumps in water. 

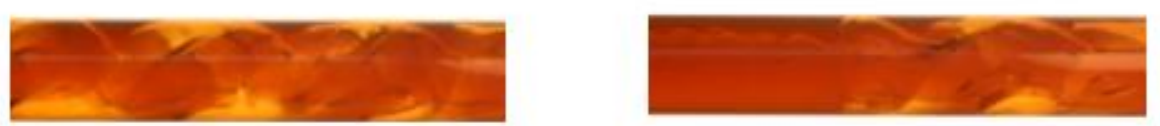

Figure 6. Pictures of the inversion flow regime. 


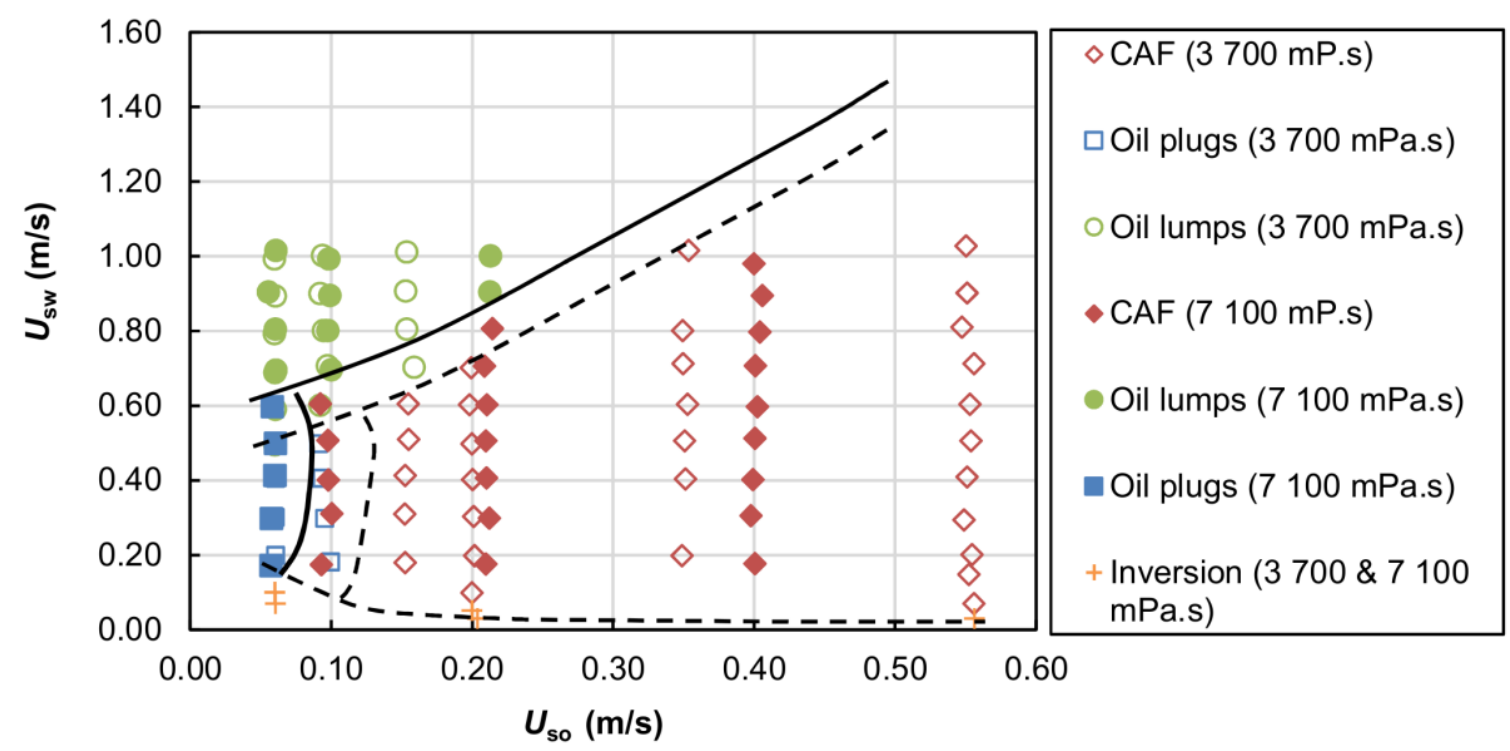

Figure 7. Experimental flow pattern maps of oil-water flow in a 1-inch pipe for oil viscosity 3300 and $7100 \mathrm{mPa} \cdot \mathrm{s}$. 


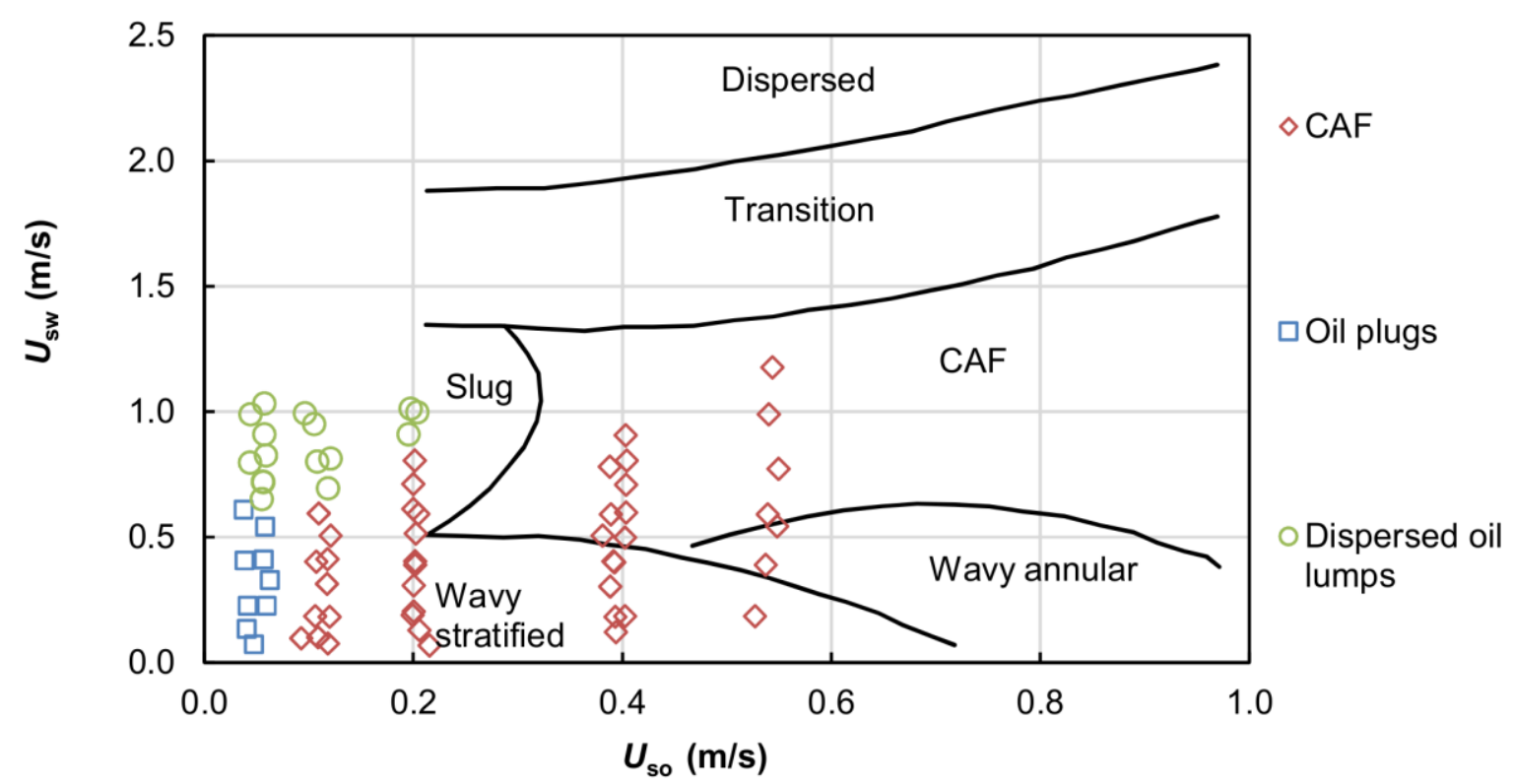

Figure 8. Comparison of flow pattern maps from the present study and Sotgia et al. ${ }^{17}$

Regimes defined in the present study are presented with markers. Regimes defined by

Sotgia et al. ${ }^{17}$ are presented with boundary lines and regime descriptions: Wavy stratified;

Wavy annular; CAF; Slug; Transition (between CAF/Slug and Dispersed); Dispersed. 


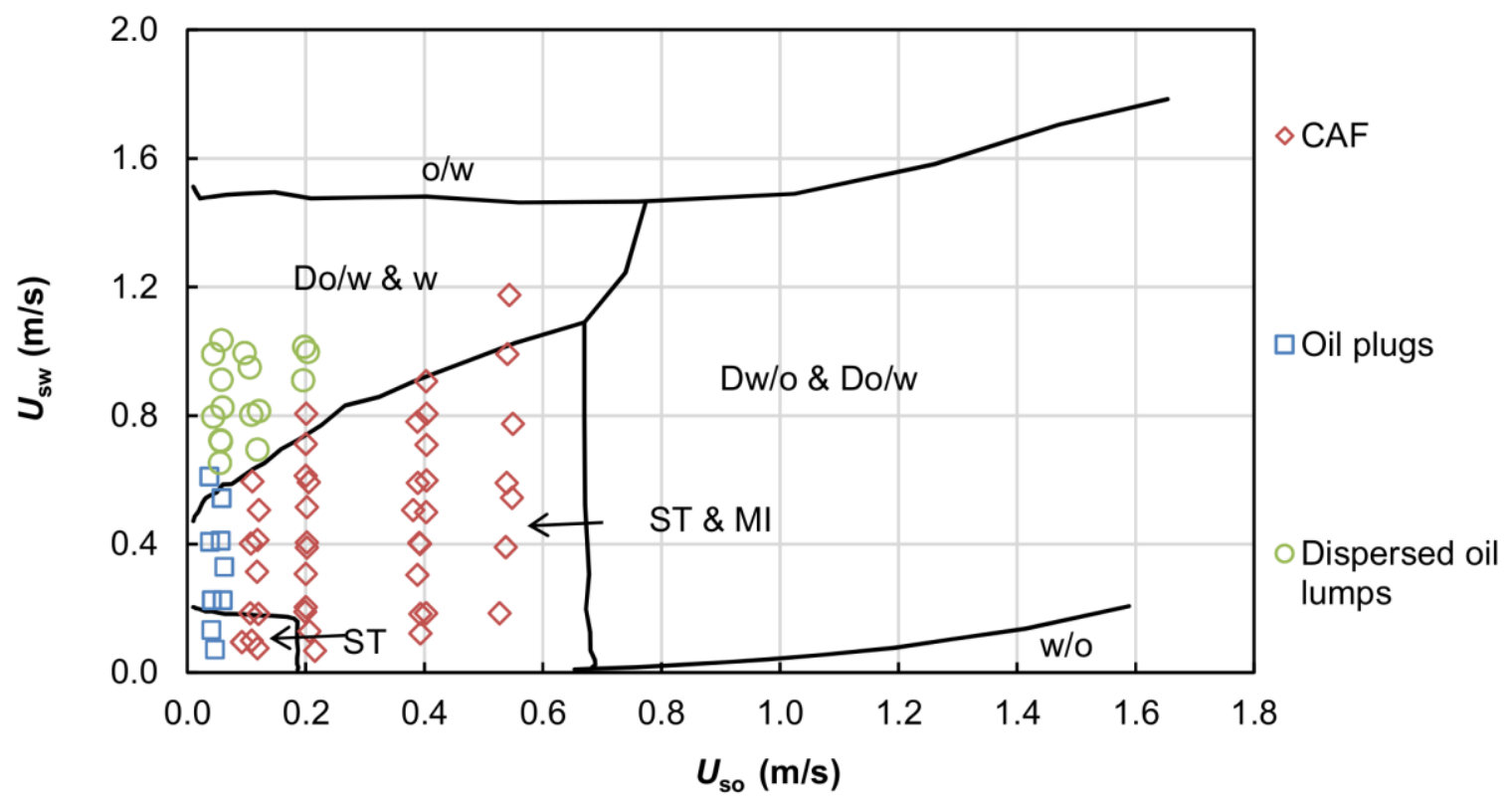

Figure 9. Comparison of flow pattern maps from the present study and Trallero et al. ${ }^{1}$

Regimes defined in the present study are presented with markers. Regimes defined by

Trallero et al. ${ }^{1}$ are presented with boundary lines and regime descriptions: Stratified (ST);

Stratified with mixing at the interface (ST \& MI); Dispersion of oil in water and water (Do/w \& w); Oil in water emulsion (o/w); Dispersion of water in oil and oil in water (Dw/o \& Do/w); Water in oil emulsion (w/o). 


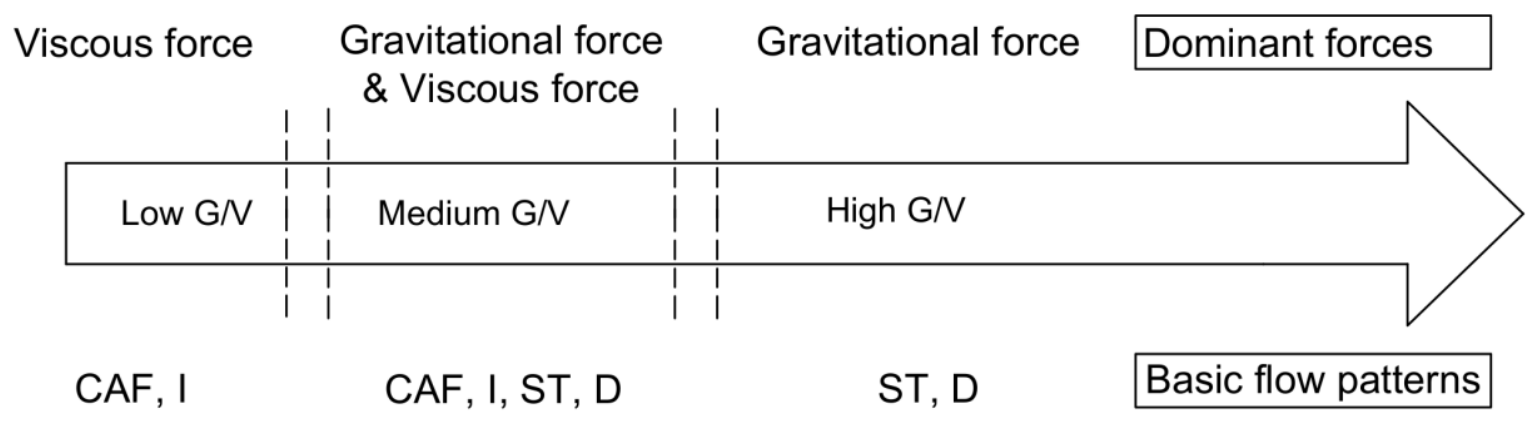

Figure 10. A diagram describing liquid-liquid flows characterized with different basic flow patterns and the corresponding gravitation to viscous force ratio (G/V). 


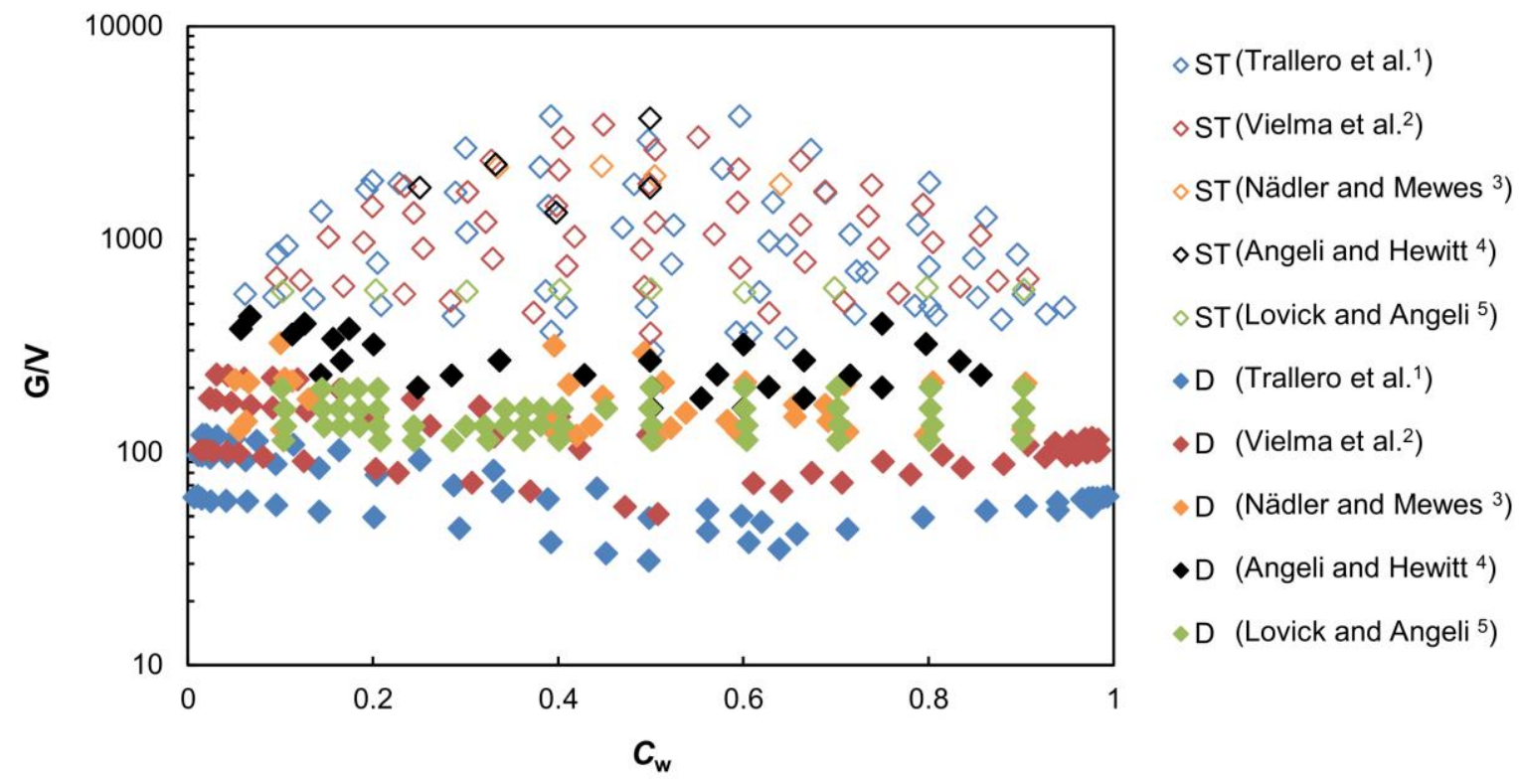

Figure 11. Basic flow patterns developed with gravitation to viscous force ratio (G/V) and $\mathrm{C}_{\mathrm{w}}$ in gravitational force dominant oil-water flows $\left(\mathrm{S}_{\mathrm{G}}\right)$. 


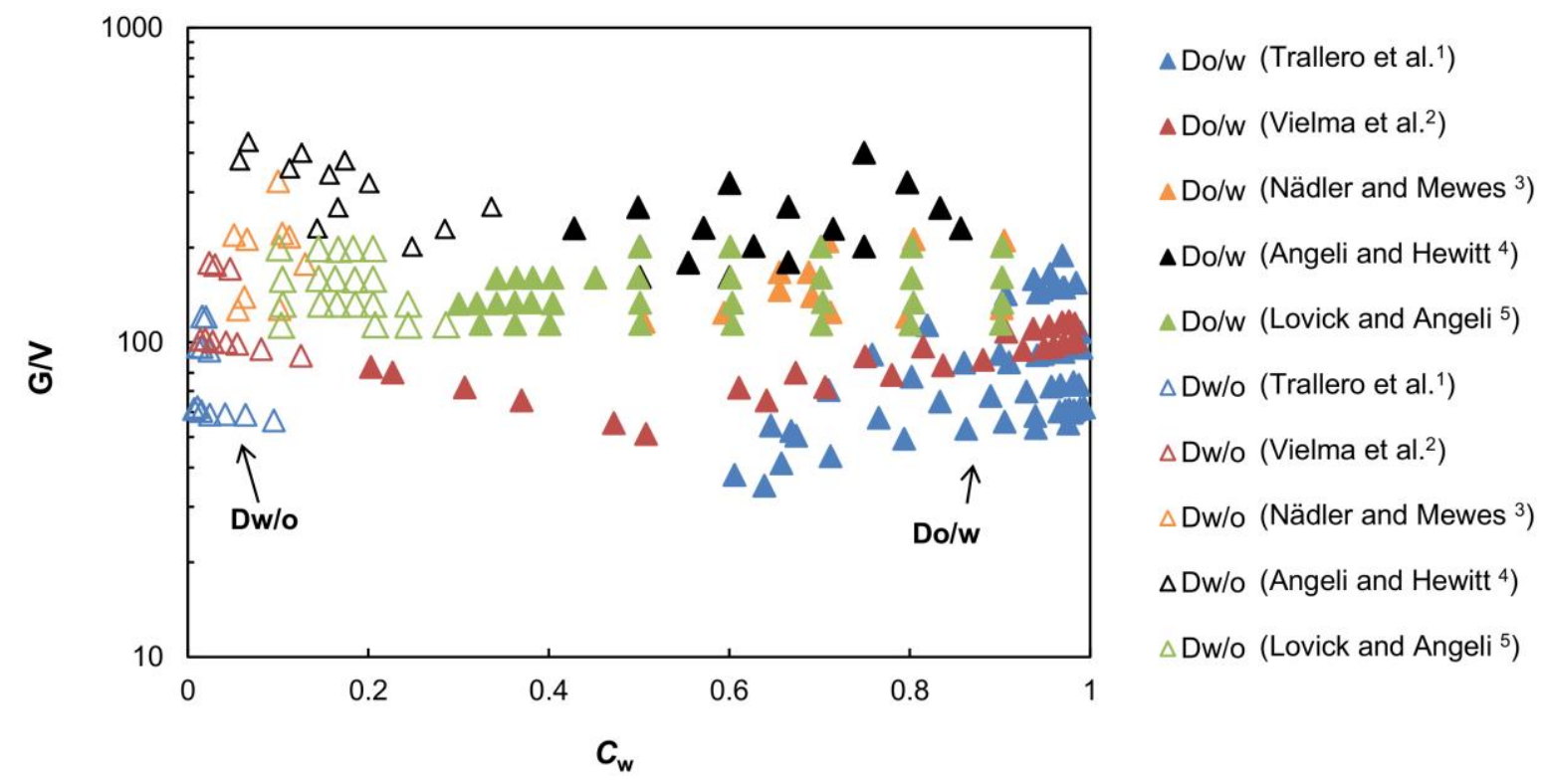

Figure 12. Specific dispersed flow patterns with gravitation to viscous force ratio $(\mathrm{G} / \mathrm{V})$ and $\mathrm{C}_{\mathrm{w}}$. 


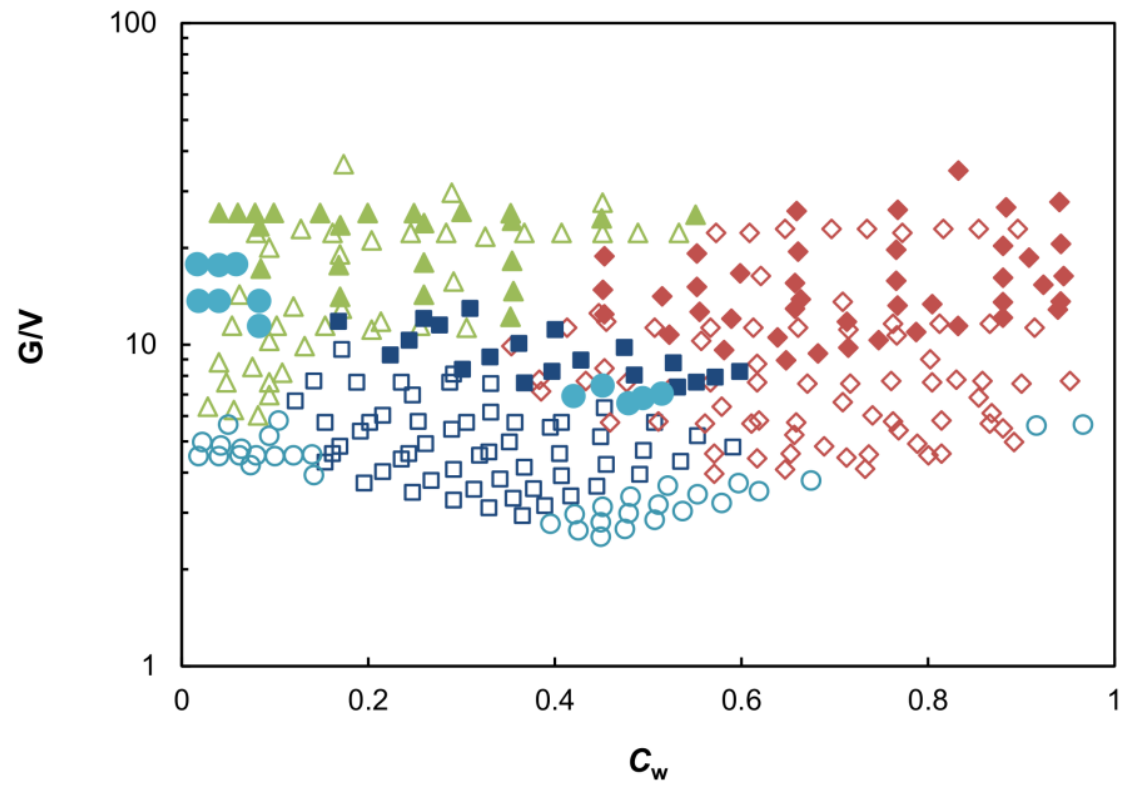

Wegmann and Rudolf von Rohr $^{8}$

$\triangle \mathrm{ST}(\mathrm{I} . \mathrm{D} .=5.6 \mathrm{~mm})$

口CAF (I.D. $=5.6 \mathrm{~mm})$

$\diamond \mid(I . D .=5.6 \mathrm{~mm})$

OD (I.D. $=5.6 \mathrm{~mm})$

$\triangle \mathrm{ST}$ (I.D. $=7 \mathrm{~mm})$

- CAF (I.D.=7 mm)

\& (I.D.=7 mm)

- $D$ (I.D.=7 mm)

Figure 13. Basic flow patterns developed with gravitation to viscous force ratio (G/V) and $\mathrm{C}_{\mathrm{w}}$ in gravitational force and viscous force comparable oil-water flows $\left(\mathrm{S}_{\mathrm{Gv}}\right)$. 


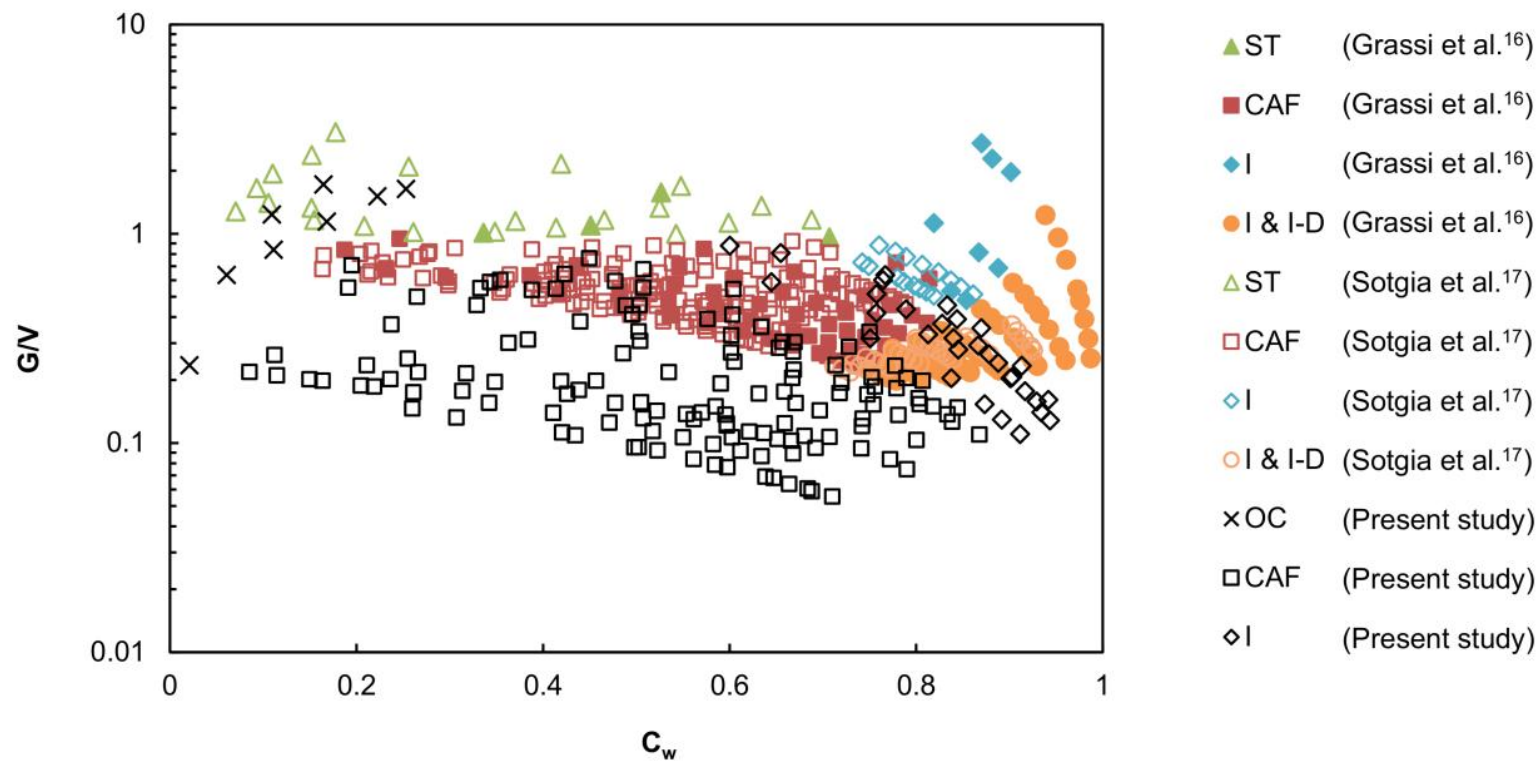

Figure 14. Basic flow patterns developed with gravitation to viscous force ratio (G/V) and $\mathrm{C}_{\mathrm{w}}$ in mainly viscous force dominant oil-water flows $\left(\mathrm{S}_{\mathrm{v}}\right)$. 


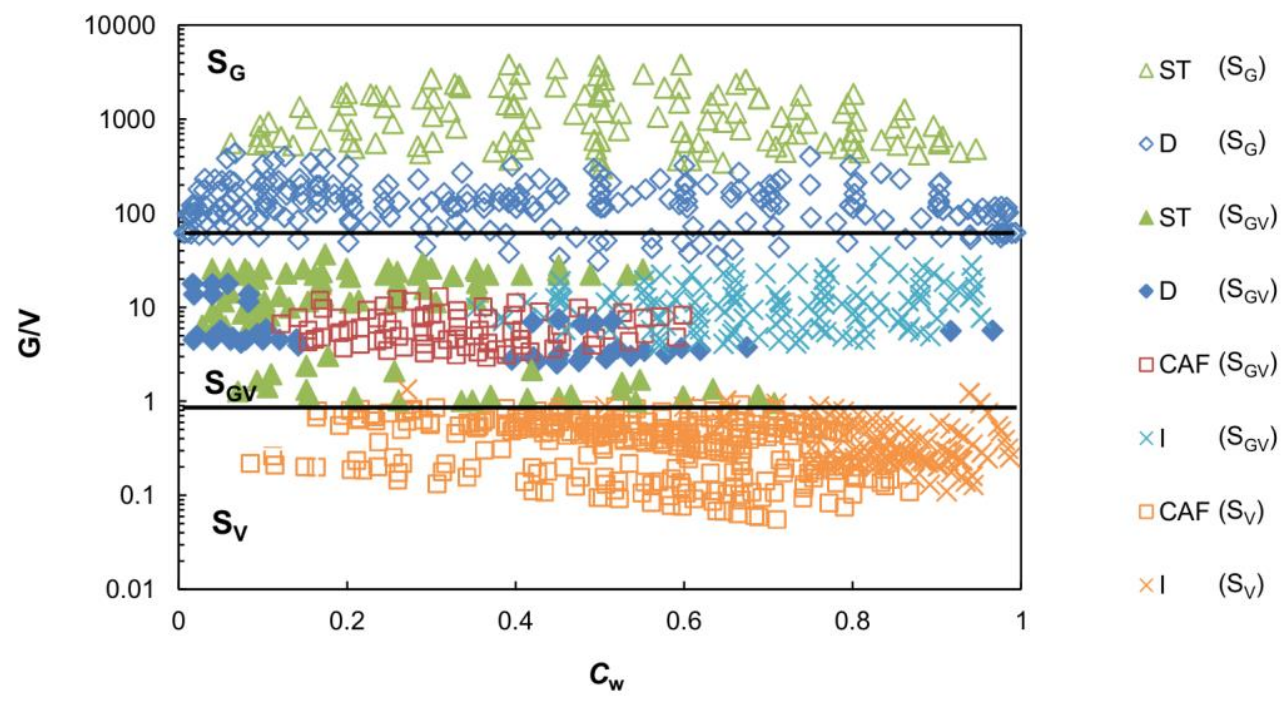

Figure 15. Basic flow patterns developed with gravitation to viscous force ratio (G/V) and $C_{w}$ in two-phase oil-water flows, $S_{G}, S_{v}$ and $S_{G v}$. Source data: Trallero et al. ${ }^{1}$, Vielma et al. ${ }^{2}$, Nädler and Mewes ${ }^{3}$, Angeli and Hewitt ${ }^{4}$, Lovick and Angeli ${ }^{5}$, Wegmann and Rudolf von Rohr ${ }^{8}$, Grassi et al. ${ }^{16}$, Sotgia et al. ${ }^{17}$ and present study. The bold lines represents approximate transitions between $\mathrm{S}_{\mathrm{G}}, \mathrm{S}_{\mathrm{GV}}$, and $\mathrm{S}_{\mathrm{V}}$. 
Table 1 Summary of the data sets used for flow map comparison.

\begin{tabular}{llllll}
\hline Data sources & $\begin{array}{l}\text { Pipe I.D. } \\
(\mathrm{mm})\end{array}$ & $\begin{array}{l}\mu_{o} \\
(\mathrm{mPa} \cdot \mathrm{s})\end{array}$ & $\begin{array}{l}\rho_{o} \\
\left(\mathrm{~kg} / \mathrm{m}^{3}\right)\end{array}$ & $\begin{array}{l}\sigma \\
(\mathrm{N} / \mathrm{m})\end{array}$ & $\begin{array}{l}\text { Flow rates } \\
(\mathrm{m} / \mathrm{s})\end{array}$ \\
\hline Trallero et al. $^{1}$ & 50 & 28.8 & 884 & 0.036 & $U_{\text {so:0.01 1.6; }} U_{\text {sw: }}: 0.02 \sim 1.6 ;$ \\
Sotgia et al. $^{17}$ & 26 & 919 & 889 & 0.02 & $U_{\text {so }}: 0.1 \sim 1.0 ; U_{\text {sw }}: 0.1 \sim 2.5 ;$ \\
Present study & 26 & 5000 & 910 & 0.02 & $U_{\text {so }}: 0.04 \sim 0.54 ; U_{\text {sw }}: 0.02 \sim 1.1 ;$ \\
\hline
\end{tabular}


Table 2 Summary of experimental studies on liquid-liquid flows in horizontal pipes.

\begin{tabular}{|c|c|c|c|c|c|c|c|c|}
\hline & $\begin{array}{l}\text { Pipe I.D. } \\
\text { (mm) }\end{array}$ & $\mu_{o}(\mathrm{mPa} \cdot \mathrm{s})$ & $\boldsymbol{\rho}_{\boldsymbol{o}}\left(\mathrm{kg} / \mathrm{m}^{3}\right)$ & $\sigma(\mathrm{N} / \mathrm{m})$ & $\begin{array}{l}\text { Velocity range } \\
(\mathrm{m} / \mathrm{s})\end{array}$ & $\begin{array}{l}\mathbf{E o}^{\prime} \\
\left(\frac{\Delta \rho g D^{2}}{8 \sigma}\right)\end{array}$ & $\begin{array}{l}\mathrm{G} / \mathrm{V} \\
\left(\frac{\Delta \rho g D^{2}}{\mu U}\right)\end{array}$ & $\begin{array}{l}\text { Basic flow } \\
\text { patterns } \\
\text { observed a) }\end{array}$ \\
\hline Trallero et al. ${ }^{1}$ & 50 & 28.8 & 884 & 0.036 & $\begin{array}{l}U_{\text {so }}: 0.01-1.6 \\
U_{\text {sw }}: 0.02-1.6\end{array}$ & 9.7 & $30-3800$ & ST, D \\
\hline Vielma et al. $^{2}$ & 50 & 18.8 & 859 & 0.029 & $\begin{array}{l}U_{\text {so }}: 0.03-1.75 \\
U_{\text {sw }}: 0.03-1.75\end{array}$ & 14.7 & $50-3500$ & ST, D \\
\hline Nädler and Mewes ${ }^{3}$ & 59 & $22,27,35$ & 845 & $0.04^{b}$ & $U_{m}: 0.1-1.6$ & 16.3 & $100-2400$ & ST, D \\
\hline Angeli and Hewitt ${ }^{4}$ & 24 & 1.6 & 801 & 0.017 & $U_{m}: 0.3-3.9$ & 8.2 & $150-3700$ & ST,D \\
\hline Lovick and Angeli ${ }^{5}$ & 38 & 6 & 828 & 0.04 & $U_{m}: 0.8-3$ & 7.6 & $100-600$ & ST,D \\
\hline Russell et al. ${ }^{7}$ & 20.5 & 18 & 832 & $0.04^{b}$ & $\begin{array}{l}U_{\text {so }}: 0.02-0.9 \\
U_{\text {sw }}: 0.04-1.08\end{array}$ & 2.1 & $18-700$ & ST, I, D \\
\hline Sridhar et al. ${ }^{18}$ & 52.5 & 220,1070 & 884 & 0.0304 & $\begin{array}{l}U_{\text {so }}: 0.1-1.0 \\
U_{\text {sw }}: 0.1-0.5\end{array}$ & 12.8 & $2.4-140$ & ST, CAF \\
\hline $\begin{array}{l}\text { Wegmann and } \\
\text { Rudolf von Rohr }{ }^{8}\end{array}$ & $5.6,7$ & $4.3-5.2$ & $818-821$ & 0.062 & $\begin{array}{l}U_{\text {so }}: 0.01-2.5 \\
U_{\text {sw }}: 0.01-2\end{array}$ & $0.11-0.17$ & $2.4-80$ & ST, CAF, I, D \\
\hline Grassi et al. ${ }^{16}$ & 21 & 800 & 886 & 0.05 & $\begin{array}{l}U_{\text {so }}: 0.03-0.7 \\
U_{\text {sw }}: 0.2-2.5\end{array}$ & 1.2 & $0.2-4$ & ST, CAF, I, D \\
\hline Sotgia et al. ${ }^{17}$ & $21-40$ & 919 & 889 & 0.02 & $\begin{array}{l}U_{\text {so }}: 0.1-1.0 \\
U_{\text {sw }}: 0.1-2.51\end{array}$ & $1.8-6.5$ & $0.4-50$ & ST, CAF, I, D \\
\hline Charles et al. ${ }^{6}$ & 26 & $6.29,16.8,65$ & 998 & 0.045 & $\begin{array}{l}\text { Uso: 0.02-0.9; } \\
\text { Usw:0.03-1.07 }\end{array}$ & 0 & 0 & CAF, I, D \\
\hline McKibben et al. ${ }^{13}$ & 53 & $\begin{array}{l}620-920 \\
5300-11000\end{array}$ & $\begin{array}{l}884-885 \\
971-976\end{array}$ & $\begin{array}{l}0.04^{b)} \\
0.04^{b)}\end{array}$ & $\begin{array}{l}U_{m}: 0.045 \\
U_{m}: 0.03-0.12\end{array}$ & $\begin{array}{l}9.7^{c)} \\
1.9-2.3^{c)}\end{array}$ & $\begin{array}{l}72-112 \\
0.4-4.8\end{array}$ & $\begin{array}{l}\text { ST } \\
\text { CAF, I }\end{array}$ \\
\hline McKibben et al. ${ }^{14}$ & $\begin{array}{l}53 \\
105\end{array}$ & $\begin{array}{l}5800-91600 \\
7100\end{array}$ & $\begin{array}{l}958-987 \\
958-984\end{array}$ & $\begin{array}{l}0.04^{b)} \\
0.04^{b)}\end{array}$ & $\begin{array}{l}U_{m}: 0.5-1.2 \\
U_{m}: 0.3-0.77\end{array}$ & $\begin{array}{l}0.9-3.4^{\mathrm{c})} \\
4.7-13.5^{\mathrm{c})}\end{array}$ & $\begin{array}{l}0.002-0.4 \\
0.07-2\end{array}$ & $\begin{array}{l}\text { CAF, I } \\
\text { CAF, I }\end{array}$ \\
\hline Arney et al. ${ }^{12}$ & 16 & 2700 & 989 & 0.26 & $\begin{array}{l}U_{\text {so }}: 0.14-1.16 \\
U_{\text {sw }}: 0.06-0.65\end{array}$ & 0.01 & $\begin{array}{l}0.004- \\
0.04\end{array}$ & CAF, I \\
\hline Al-Awadi ${ }^{19}$ & 26 & $3840-16000$ & $906-938$ & $0.04^{b)}$ & $U_{\text {so }}: 0.06-0.57$ & $1.2-2.0^{c)}$ & $0.01-1.6$ & CAF, I \\
\hline Alagbe $^{20}$ & 26 & $3700-7100$ & $905-920$ & 0.02 & $\begin{array}{l}U_{\text {so }}: 0.06-0.4 \\
U_{\text {sw }}: 0.2-1.0\end{array}$ & $3.2-3.8$ & $0.04-0.8$ & CAF, I \\
\hline $\mathrm{Shi}^{21}$ & 26 & $3300-16000$ & $905-938$ & 0.02 & $\begin{array}{l}U_{\text {so }}: 0.04-0.57 \\
U_{\text {sw }}: 0.01-1.18\end{array}$ & $1.2-3.8$ & $0.01-1.6$ & CAF, I \\
\hline Ooms et al. ${ }^{9}$ & $\begin{array}{l}50, \\
203.2\end{array}$ & $1200-3300$ & $953-968$ & $0.04^{b)}$ & $\begin{array}{l}U_{\text {so }}: 1.0 ; \\
U_{\text {sw: }}: 0.01-0.25\end{array}$ & $2.3-55^{c)}$ & $0.1-3.2$ & $\mathrm{CAF}^{\mathrm{d})}$ \\
\hline Oliemans et al. ${ }^{10}$ & 50 & 3000 & 978 & $0.04^{b)}$ & $\begin{array}{l}U_{\text {so }}: 0.5-1.0 \\
U_{\text {sw }}: 0.03-0.25\end{array}$ & $1.5^{c)}$ & $0.08-0.4$ & $\mathrm{CAF}^{\mathrm{d})}$ \\
\hline Bannwart ${ }^{11}$ & 22.5 & 270 & 989 & $0.04^{b)}$ & $\begin{array}{l}U_{\text {so }}: 0.30-0.63 \\
U_{\text {sw }}: 0.03-0.28\end{array}$ & $0.14^{\mathrm{c})}$ & $0.1-0.5$ & $\mathrm{CAF}^{\mathrm{d})}$ \\
\hline $\begin{array}{l}\text { Rodriguez and } \\
\text { Baldani } 22\end{array}$ & 26 & 280 & 828 & 0.034 & $\begin{array}{l}U_{\text {so }}: 0.03-0.15 \\
U_{\text {sw }}: 0.1-0.15\end{array}$ & 4 & $12-32$ & ST e) \\
\hline
\end{tabular}

a) Different nomenclatures are used by different authors. Only the basic flow patterns, namely ST, CAF, I and D, are listed.

b) The interfacial tension is not reported; a medium value of 0.04 is used here as an estimate to calculate the corresponding $\mathrm{Eo}^{\prime}$.

c) The Eo' is obtained with an estimated interfacial tension of 0.04 .

d) This experiment specifically focuses on core annular flow thus no other flow patterns are reported.

e) This experiment specifically focuses on stratified flow thus no other flow patterns are reported. 
